Check for updates

Cite this: RSC Adv., 2019, 9, 30964

Received 17th May 2019

Accepted 18th August 2019

DOI: $10.1039 / c 9 r a 03735 g$

rsc.li/rsc-advances

\section{Theoretical predictions for low-temperature phases, softening of phonons and elastic stiffnesses, and electronic properties of sodium peroxide under high pressure $\uparrow$}

\author{
Pornmongkol Jimlim, (D) abc Prutthipong Tsuppayakorn-aek, ${ }^{\text {ab }}$ \\ Teerachote Pakornchote, ${ }^{\text {ab }}$ Annop Ektarawong, ${ }^{\text {ab }}$ Udomsilp Pinsook (D) ab \\ and Thiti Bovornratanaraks (D) *ab
}

\begin{abstract}
High-pressure phase stabilities up to $600 \mathrm{~K}$ and the related properties of $\mathrm{Na}_{2} \mathrm{O}_{2}$ under pressures up to $300 \mathrm{GPa}$ were investigated using first-principles calculations and the quasi-harmonic approximation. Two high-pressure phases of $\mathrm{Na}_{2} \mathrm{O}_{2}$ that are thermodynamically and dynamically stable were predicted consisting of the Amm2 (distorted $P \overline{6} 2 \mathrm{~m}$ ) and the $P 2_{1} / c$ structures, which are stable at low temperature in the pressure range of $0-22 \mathrm{GPa}$ and $22-28 \mathrm{GPa}$, respectively. However, the $P \overline{6} 2 m$ and Pbam structures become the most stable instead of the Amm2 and $P 2_{1} / c$ structures at the elevated temperatures, respectively. Interestingly, the softening of some phonon modes and the decreasing of some elastic stiffnesses in the Amm2 structure were also predicted in the pressure ranges of 2-3 GPa and 9-10 GPa. This leads to the decreasing of phonon free energy and the increasing of the ELF value in the same pressure ranges. The HSE06 band gaps suggest that all phases are insulators, and they increase with increasing pressure. Our findings provide the $P-T$ phase diagram of $\mathrm{Na}_{2} \mathrm{O}_{2}$, which may be useful for investigating the thermodynamic properties and experimental verification.
\end{abstract}

\section{Introduction}

Sodium peroxide $\left(\mathrm{Na}_{2} \mathrm{O}_{2}\right)$ is of great interest because of its broad applications, for example as a bleaching agent, oxidizing agent, and in breathing apparatus. ${ }^{\mathbf{1 , 2}} \mathrm{Na}_{2} \mathrm{O}_{2}$ is also utilized as an oxygen source by capturing carbon dioxide to generate oxygen and sodium carbonate. ${ }^{1}$ Moreover, $\mathrm{Na}_{2} \mathrm{O}_{2}$ has attracted much attention in energy storage for $\mathrm{Na}-\mathrm{O}_{2}$ batteries due to its high energy density. ${ }^{3}$ However, the formation of a discharge product of $\mathrm{Na}_{2} \mathrm{O}_{2}$ on the cathode of the batteries leads to poor reversibility and increasing overpotential during recharge. ${ }^{3}$ Therefore,

${ }^{a}$ Extreme Conditions Physics Research Laboratory, Physics of Energy Materials Research Unit, Department of Physics, Faculty of Science, Chulalongkorn University, Bangkok 10330, Thailand. E-mail: thiti.b@chula.ac.th; Fax: +66 2253 1150; Tel: +66 22187554

${ }^{b}$ Thailand Center of Excellence in Physics, Commission on Higher Education, Bangkok 10400, Thailand

${ }^{c}$ Department of Physics, Mahidol Wittayanusorn School, Nakhon Pathom 73170, Thailand

$\dagger$ Electronic supplementary information (ESI) available: Structural data; bulk and shear moduli; vibrational modes, activity in the IR and Raman spectra; comparison of $E, H$ and $P V$ between the Amm2 and the $P 2 m$ structures; phonon dispersions curves; lattice constants versus pressure; peroxide group alignments; interatomic distances versus pressure; the universal elastic anisotropy index versus pressure; the relative Gibbs free energy versus temperature; the phonon free energy versus volume. See DOI: 10.1039/c9ra03735g obtaining in-depth understanding about the structural and thermodynamic stabilities of $\mathrm{Na}_{2} \mathrm{O}_{2}$ is of fundamental importance for improving the performance of $\mathrm{Na}-\mathrm{O}_{2}$ batteries.

It is well-known that pressure can be employed to synthesize novel materials with different electronic structures, properties, and stoichiometries. ${ }^{4}$ Consequently, an in-depth understanding of the structural phase transitions and related properties (e.g. elastic, vibrational, and electronic properties) of $\mathrm{Na}_{2} \mathrm{O}_{2}$ under high pressure is paramount. Previously, the crystal structure of $\mathrm{Na}_{2} \mathrm{O}_{2}$ at ambient conditions has been investigated. Tallman et al. and Föppl independently studied this at room temperature using single-crystal/powder X-ray diffraction measurements. They found that the crystal structure of $\mathrm{Na}_{2} \mathrm{O}_{2}$ is hexagonal with the $P \overline{6} 2 m$ space group and lattice constants of $a=6.22 \AA$ and $c=$ $4.47 \AA$ were reported by Tallman et $a l .{ }^{5}$ and of $a=6.208 \AA$ and $c=4.469 \AA$ were reported by Föppl $^{6}$ (see Table S1, ESI $\dagger$ ). However, although the lattice constants of Tallman's and Föppl's structures are not significantly different within the maximal error of $\pm 0.01 \AA$, the atomic positions of $\mathrm{Na} 1$ and $\mathrm{Na} 2$ are significantly different with atomic displacements of $0.118 \AA$ and $0.012 \AA$, respectively. Thus, the most stable structure at room temperature should be identified theoretically. Very recently, Dunst et al. ${ }^{7}$ used the Rietveld refinement methods to analyze the $\mathrm{X}$-ray powder diffraction patterns of microcrystalline and nanocrystalline $\mathrm{Na}_{2} \mathrm{O}_{2}$. The obtained structures were 
consistent with Föppl's structure. In addition, Tallman et al. ${ }^{8}$ reported evidence for two new phases of $\mathrm{Na}_{2} \mathrm{O}_{2}$ that were observed over the range of $25-550{ }^{\circ} \mathrm{C}$. One was found at $512{ }^{\circ} \mathrm{C}$, and the other was found at room temperature and was prepared by pouring liquid $\mathrm{Na}_{2} \mathrm{O}_{2}$ into liquid air. ${ }^{8,9}$ They suggested that the quenched $\mathrm{Na}_{2} \mathrm{O}_{2}$ may be stable in the temperature range of 596-675 ${ }^{\circ} \mathrm{C}$ or at low temperature. ${ }^{8}$ However, the crystal structures of the two phases have not been identified. Therefore, the determination of new phase structures of $\mathrm{Na}_{2} \mathrm{O}_{2}$ at room and high temperature needs guidance from theoretical prediction.

As for previous high-pressure study, Deng et al. ${ }^{\mathbf{1 0}}$ investigated the structural phase transition of $\mathrm{Na}_{2} \mathrm{O}_{2}$ in the range of 0 $100 \mathrm{GPa}$ using first-principles calculations. They employed the particle swarm optimization algorithm (CALYPSO) to search the lowest enthalpy structures at given pressures and optimized them using the projected augmented-wave potentials with $3 \mathrm{~s}^{1} \mathrm{p}^{0}$ and $2 \mathrm{~s}^{2} 2 \mathrm{p}^{4}$ as valence electrons for $\mathrm{Na}$ and $\mathrm{O}$, respectively, and the Perdew-Burke-Ernzerhof (PBE) functional as implemented in the Vienna $A b$ initio Simulation Package (VASP). They predicted that the hexagonal $P \overline{6} 2 m$ structure transforms to the orthorhombic Pbam structure at around $28 \mathrm{GPa} .^{\mathbf{1 0}}$ However, since the high-pressure phases of $\mathrm{Na}_{2} \mathrm{O}_{2}$ are waiting for experimental confirmation, the structural phase transition is an open question for further theoretical prediction, which has been conducted in the present work.

In this work, the first-principles calculations based on the density functional theory (DFT) formalism and the quasiharmonic approximation (QHA) were employed to investigate thermodynamic phase stabilities in order to find new structures of $\mathrm{Na}_{2} \mathrm{O}_{2}$ at high pressures. Here the ab initio random structure searching (AIRSS) method was used to search the lowest enthalpy structures. Moreover, the structural phase transition in $\mathrm{Na}_{2} \mathrm{O}_{2}$ was also reviewed and extended up to $300 \mathrm{GPa}$ based on the DFT calculations. The elastic, vibrational, and electronic properties of $\mathrm{Na}_{2} \mathrm{O}_{2}$ at high pressures were also calculated to describe their properties. In addition, the VESTA program was also employed to visualize all crystal structures. ${ }^{11}$

\section{Computational methods}

All calculations were performed using the Cambridge Serial Total Energy Package (CASTEP), ${ }^{12}$ which is based on DFT calculations. Ultrasoft pseudopotentials ${ }^{13}$ were generated for Na: $2 s^{2} 2 p^{6} 3 s^{1}$ and O: $2 s^{2} 2 p^{4}$, and the GGA-PBE exchangecorrelation functional ${ }^{14}$ was employed with a plane-wave basis cutoff of $760 \mathrm{eV}$ and a $k$-point grid spacing of $2 \pi \times 0.04 \AA^{-1}$. In order to optimize the unit cells, the ultrafine quality of convergence tolerance was set as follows: energy change less than $1.0 \times 10^{-6} \mathrm{eV}$ atom $^{-1}$, a residual force less than $0.01 \mathrm{eV}$ $\AA^{-1}$, atomic displacement within $5.0 \times 10^{-4} \AA$, and stress below $0.02 \mathrm{GPa}$. Furthermore, to calculate the phonon and electronic properties, the cells were optimized again with a denser grid spacing of $2 \pi \times 0.02 \AA^{-1}$ and maximum force less than $0.001 \mathrm{eV}$ $\AA^{-1}$.

In order to search the lowest enthalpy structure in the pressure range of $0-300 \mathrm{GPa}$, the AIRSS technique ${ }^{15}$ was performed at $0,10,20,30,100,200$, and $300 \mathrm{GPa}$ by generating the random cells that contained $\mathrm{Na}_{2} \mathrm{O}_{2}$ with $1,2,3,4$, and 6 formula units (f.u.). Furthermore, the random atomic displacements and random unit cell deformations (namely "shaking") ${ }^{4}$ were also used to generate the mutated structures of the Tallman's and the Föppl's structures at ambient pressure. By generating initial structures with different lattice parameters and number of formula units that implemented reasonable bond lengths and unit cell volumes at each pressure, the structures were relaxed to the enthalpy minimum and then the lowest enthalpy structure selected for refining the structures and their energies. ${ }^{15,16}$ The simple linear approximation was employed to estimate the enthalpies $(H)$ of structures at given pressures:

$$
H(P) \simeq H\left(P_{\mathrm{s}}\right)+\left(P-P_{\mathrm{s}}\right) V_{\mathrm{s}},
$$

where $H\left(P_{\mathrm{s}}\right)$ is the lowest enthalpy of the most stable structure at pressure $P_{\mathrm{s}}$ with volume $V_{\mathrm{s}}$, while $H(P)$ is the enthalpy of different structures which may become more stable at another pressure $P .{ }^{15}$ To obtain the pressure dependence of enthalpy for the lowest enthalpy structure, the total energy versus unit cell volume relations were fitted using the third-order Birch-Murnaghan equations of states, ${ }^{17}$ and the enthalpies at the pressures from 0 to $300 \mathrm{GPa}$ were calculated using the formula $H=$ $E+P V$.

Elastic constants calculations were performed to analyze the evolution of the elastic constants, bulk and shear moduli, and elastic anisotropy of $\mathrm{Na}_{2} \mathrm{O}_{2}$ for the ambient phase at elevated pressure. By generating the set of distorted structures that is required by the symmetry, the full $6 \times 6$ tensor of elastic constants was produced to determine the stress-strain relationships. ${ }^{18}$ Furthermore, the universal elastic anisotropy index $\left(A^{\mathrm{U}}\right)$ was used to quantify the elastic anisotropy, which was obtained from the formula ${ }^{19}$

$$
A^{\mathrm{U}}=\frac{K^{\mathrm{V}}}{K^{\mathrm{R}}}+5 \frac{G^{\mathrm{V}}}{G^{\mathrm{R}}}-6,
$$

where the superscripts $\mathrm{R}$ and $\mathrm{V}$ represent the lower (Reuss) and upper (Voigt) bounds on the bulk $(K)$ and shear moduli $(G)$, respectively. Reuss and Voigt of the bulk $\left(K^{\mathrm{R}}, K^{\mathrm{V}}\right)$ and shear moduli $\left(G^{\mathrm{R}}, G^{\mathrm{V}}\right)$ were calculated from the elastic constants using the Voigt-Reuss-Hill approximations. ${ }^{20}$

To verify the dynamical stability of the predicted structures, phonon dispersions and phonon density of states were calculated using the finite-displacement supercell method. ${ }^{21}$ Moreover, the phase stabilities of the predicted structures under different pressures and temperatures were also verified by comparing the differences of Gibbs free energies $(\Delta G)$ between those structures. The Gibbs free energies, $G(P, T)$, as a function of pressure and temperature were calculated using the standard thermodynamic relations $G=F+P V$, and the thermodynamic calculations were conducted based on the QHA. ${ }^{22}$ Helmholtz free energies, $F(V, T)$, were calculated as

$$
\begin{gathered}
F(V, T)=E(V)+F_{\mathrm{ph}}(V, T), \\
F_{\mathrm{ph}}(V, T)=k_{\mathrm{B}} T \int_{0}^{\infty} g(\omega) \ln \left[2 \sinh \left(\frac{\hbar \omega}{2 k_{\mathrm{B}} T}\right)\right] \mathrm{d} \omega,
\end{gathered}
$$


where $E(V)$ is the ground-state total energy at $0 \mathrm{~K}$ as a function of volume, and $F_{\mathrm{ph}}(V, T)$ represents the phonon free energy at volume $V$ as a function of temperature $T$ including the zeropoint vibrational energy. $\omega=\omega(V)$ stands for the volume dependence of phonon frequencies, and $g(\omega)$ is the phonon density of states. $h$ and $k_{\mathrm{B}}$ are the reduced Planck's and Boltzmann's constants, respectively. ${ }^{23}$ Thus, $G(P, T)$ can be rewritten as follows:

$$
\begin{gathered}
G(P, T)=E(V)+F_{\mathrm{ph}}(V, T)+P(V, T) V, \\
P(V, T)=-\frac{\partial F(V, T)}{\partial V}=P(V)-\frac{\partial F_{\mathrm{ph}}(V, T)}{\partial V}, \\
G(P, T)=E(V)+F_{\mathrm{ph}}(V, T)+P(V) V-V \frac{\partial F_{\mathrm{ph}}(V, T)}{\partial V} .
\end{gathered}
$$

In eqn (7), $E(V)$ and $P(V)$ were obtained from the DFT calculations that were fitted using the third-order Birch-Murnaghan equations of states and $F_{\mathrm{ph}}(V, T)$ was obtained from eqn (4). The relationships between $F_{\mathrm{ph}}(V, T)$ and $V$ for various volumes at the same temperature were obtained from using the third-order polynomial fitting and then calculating the last term of $\partial F_{\mathrm{ph}}(-$ $V, T) / \partial V$ at various temperatures $T$. Finally, the relationships between $G(P, T)$ and $P$ at the same temperatures were also fitted using the third-order polynomial function in order to calculate the $G(P, T)$ for each structure at given pressures and temperatures.

In order to investigate the electronic properties and bonding in the predicted structures of $\mathrm{Na}_{2} \mathrm{O}_{2}$ at high pressures, the electronic band structures and the partial density of states (PDOSs) were determined using the $\mathrm{PBE}^{14}$ and HSE06 ${ }^{24}$ functionals. The HSE06 band structures and their PDOSs were calculated using the relaxed structures obtained from the PBE functional. Furthermore, ELFs were also calculated to describe the pressure effect on the chemical bonding in the Amm2 phase of $\mathrm{Na}_{2} \mathrm{O}_{2}$.

\section{Results and discussion}

\subsection{Structural phase transitions and phase stabilities}

3.1.1 High-pressure structural phase transitions at $0 \mathrm{~K}$. By employing the AIRSS search for $\mathrm{Na}_{2} \mathrm{O}_{2}$ structures in the pressure range of $0-300 \mathrm{GPa}$, the findings at 0,10 , and $20 \mathrm{GPa}$ revealed the two stable structures consisting of the hexagonal $P \overline{6} 2 \mathrm{~m}$ structure ( 3 f.u. per cell) and the orthorhombic Amm 2 structure (6 f.u. per cell), when their enthalpy difference is only $2 \mathrm{meV}$ f.u. ${ }^{-1}$ (Fig. 1). However, the Amm2 structure is a slightly distorted version of the $P \overline{6} 2 \mathrm{~m}$ structure because the $P \overline{6} 2 \mathrm{~m}$ structure can be relaxed to be the Amm2 structure, and their simulated Xray diffraction (XRD) patterns are slightly different (Fig. S1(a), ESI $\dagger$ ). Moreover, the two crystal structures which are the monoclinic $P 22_{1} / c$ structure ( $2 \mathrm{f}$.u. per cell) and the orthorhombic Pbam structure (2 f.u. per cell) are also found at $30 \mathrm{GPa}$ (Fig. 1). The enthalpy difference between the $P 2_{1} / c$ and Pbam structures is small. Nevertheless, the XRD patterns of both structures are significantly different (Fig. S1(b) $\dagger$ ). Furthermore, the $P 2_{1} / c$
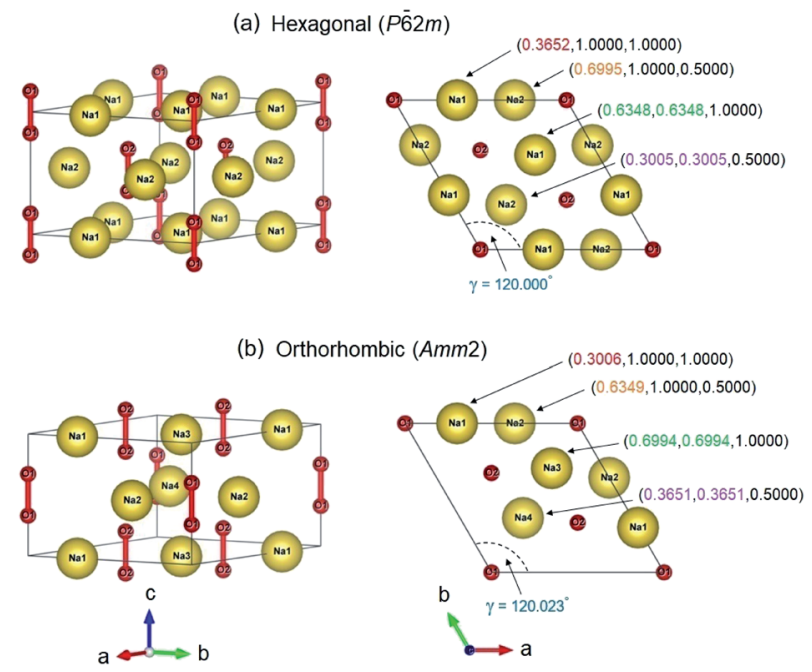

(c) Monoclinic $\left(P 2_{1} / c\right)$

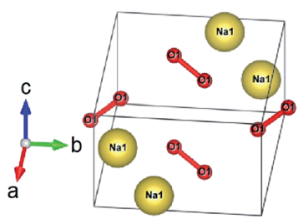

(d) Orthorhombic (Pbam)

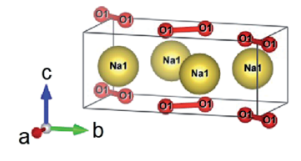

Fig. 1 The crystal structures: ( $a$ and $b$ ) the $P 62 m$ and the primitive Amm2 structures viewed along two directions at ambient pressure. The mapping of atomic positions for the $\mathrm{Na}$ atoms marked by the same color. (c) The $P 2_{1} / c$ structure at $22 \mathrm{GPa}$, and (d) the Pbam structure at $28 \mathrm{GPa}$.

structure is dynamically stable at $30 \mathrm{GPa}$ but unstable at $40 \mathrm{GPa}$, while the Pbam structure is dynamically stable at 30-300 GPa (Fig. S2, ESI $\dagger$ ). Here the Pbam structure is same as the structure proposed by Deng et al. ${ }^{10}$ when the structure is shifted to $(0,0,1 /$ 2).

Moreover, the AIRSS results at ambient pressure revealed that the simulated XRD patterns of the structures look like the XRD patterns calculated from the experimental results of Tallman et al. ${ }^{8}$ One is the $14 / \mathrm{mmm}$ structure which is approximate to the $\mathrm{Na}_{2} \mathrm{O}_{2}$-II at $550{ }^{\circ} \mathrm{C}$ (Fig. S3, ESI $\dagger$ ), and the other structures $\left(\mathrm{C} 2 / \mathrm{m}, \mathrm{Pmmm}\right.$, and Immm) are close to the $\mathrm{Na}_{2} \mathrm{O}_{2}-\mathrm{Q}$ at room temperature (Fig. S4, ESI $\dagger$ ). The $14 / \mathrm{mmm}$ structure is higher enthalpy than the $P \overline{6} 2 m$ structure by $0.560 \mathrm{eV} \mathrm{f.u.}{ }^{-1}$, while the $\mathrm{C} 2 / \mathrm{m}$, Pmmm, and Immm structures are higher enthalpy than the $P \overline{6} 2 m$ structure by $0.018,0.019$, and $0.021 \mathrm{eV} \mathrm{f.u.}{ }^{-1}$, respectively. With increasing pressure, these structures have increasing trends of enthalpy with respect to the $P \overline{6} 2 \mathrm{~m}$ structure in the pressure range of $0-22 \mathrm{GPa}$, and they have increasing trends of enthalpy with respect to the $P 2_{1} / c$ and Pbam structures in the pressure range of $22-100 \mathrm{GPa}$ as well (Fig. S5, ESI†). This suggests that these structures become more unstable with elevated pressure. Therefore, at high temperature below the melting point of $948 \mathrm{~K}$, the $14 / \mathrm{mmm}$ structure might be a candidate for the $\mathrm{Na}_{2} \mathrm{O}_{2}$-II phase, whereas the $C 2 / m, P m m m$, and Immm structures might be candidates for the $\mathrm{Na}_{2} \mathrm{O}_{2}-\mathrm{Q}$ phase. Generally, the symmetry of the crystal structure can be 
increased by increasing the temperature. Thus, the Immm structure may become stable at a high temperature as it is higher symmetry than the other structures.

Using geometry optimization, the Amm2 structure at $0 \mathrm{GPa}$ has an energy lower than that of the $P \overline{6} 2 \mathrm{~m}$ structure by 2.1 and 0.4 meV f.u. ${ }^{-1}$ calculated by the GGA-PBE ${ }^{14}$ and $\mathrm{LDA}^{25,26}$ functionals, respectively. These energies are slightly significant within the tolerance of the calculations. Herein the PBE functional was selected to quantify the energy difference between the Amm 2 and $P \overline{6} 2 m$ structures. Furthermore, the relaxed lattice parameters for the $P \overline{6} 2 m$ structure are in agreement with previous studies: $:^{2,5,6,27} a=b=6.282 \AA, c=4.511 \AA$, and $\gamma=$ $120.000^{\circ}$. The Amm 2 structure can be reduced to a primitive cell with the relaxed lattice parameters, $a=b=6.284 \AA$, $c=4.510 \AA$, and $\gamma=120.023^{\circ}$, which are slightly different from those of the $P \overline{6} 2 m$ structure (Fig. 1). These lattice parameters also support that the $A m m 2$ structure is the distorted $P \overline{6} 2 m$ structure. Moreover, we found that there are angle differences in the same hexagonal Na layer between the $2 \times 2 \times 2$ supercell of the primitive $A m m 2$ and the $P \overline{6} 2 m$ structures that were verified dynamically. The angles in the hexagonal Na layer of the $P \overline{6} 2 \mathrm{~m}$ structure are $60.000^{\circ}, 55.031^{\circ}, 60.000^{\circ}, 55.031^{\circ}, 60.000^{\circ}$, and $69.938^{\circ}$ as marked by $\theta 1, \theta 2, \theta 3, \theta 4, \theta 5$, and $\theta 6$, respectively (Fig. S6(a), ESI $\dagger$ ). These angles indicate that the $P \overline{6} 2 \mathrm{~m}$ structure has the distorted close-packed Na layers. However, these angles are slightly different from that of the primitive Amm 2 structure. The angles of the Amm 2 structure are $59.995^{\circ}, 55.060^{\circ}, 60.003^{\circ}$, $55.059^{\circ}, 60.003^{\circ}$, and $69.881^{\circ}$ as marked by $\theta 1, \theta 2, \theta 3, \theta 4, \theta 5$, and $\theta 6$, respectively (Fig. S6(b), ESI $\dagger$ ). In addition, these angles have three trends of change with elevated pressure consisting of an increase of $\theta 6$, a decrease of $\theta 2$ and $\theta 4$, and no change in $\theta 1$, $\theta 3$, and $\theta 5$. The differences in these angles between both structures at the same pressures are shown in Fig. S7, ESI. $\dagger$ This suggests that the primitive Amm2 structure is a more distorted close-packed Na layer than the $P \overline{6} 2 m$ structure (deviation from $60^{\circ}$ ). These suggest that the Amm2 (distorted $P \overline{6} 2 \mathrm{~m}$ ) structure is slightly different to the $P \overline{6} 2 m$ structure. However, this results in significant differences in their phonon dispersions and their electronic band structures, which will be discussed, respectively. Consequently, it is possible that the $\mathrm{Na}_{2} \mathrm{O}_{2}$ phase at extremely low temperature favors the lower symmetry (Amm2, No. 38) rather than the higher symmetry ( $P \overline{6} 2 m$, No. 189).

Moreover, Fig. 2 also shows differences in the phonon dispersion and the partial phonon density of states between the primitive $A m m 2$ and the $P \overline{6} 2 m$ structures at ambient pressure. At the $\Gamma$ point, the phonon frequencies of the Amm 2 structure at $\sim 23.3$ and $\sim 23.5 \mathrm{THz}$ have higher values than those of the $P \overline{6} 2 \mathrm{~m}$ structure, of about 5 and $4 \mathrm{~cm}^{-1}$, respectively, while the highest frequency of both structures are slightly different. Because these phonon frequencies directly relate to the $\mathrm{O}-\mathrm{O}$ stretching modes, they suggest that the $\mathrm{O}-\mathrm{O}$ bonding in the Amm2 structure at ambient pressure is stronger than that of the $P \overline{6} 2 m$ structure. Furthermore, the phonon frequencies below $5 \mathrm{THz}$ at the $\Gamma$, Y and S points of the Amm2 structure are less than those of the $P \overline{6} 2 m$ structure at the $\Gamma, \mathrm{K}$, and $\mathbf{M}$ points, respectively. Although both structures were different, there was no imaginary phonon frequency in both curves indicating the dynamical stability of the Amm2 and $P \overline{6} 2 m$ structures at $0 \mathrm{~K}$. Moreover, as there were several different phonon frequencies, it is possible to have different Helmholtz free energies at elevated temperature.

In addition, the formation enthalpy of $\mathrm{Na}_{2} \mathrm{O}_{2}, \mathrm{Na}_{2} \mathrm{O}$, and $\mathrm{NaO}_{2}$ compounds at $0 \mathrm{~K}$ and the selected pressures of $0,10,20$, 30 , and $50 \mathrm{GPa}$ were calculated to verify the phase stability of $\mathrm{Na}_{2} \mathrm{O}_{2}$ using the formula: $\Delta H_{\mathrm{f}}=\left[H\left(\mathrm{Na}_{x} \mathrm{O}_{y}\right)-x H(\mathrm{Na})-y H(\mathrm{O})\right] /$ $(x+y)$, where $H\left(\mathrm{Na}_{x} \mathrm{O}_{y}\right)$ is the enthalpy of the considered

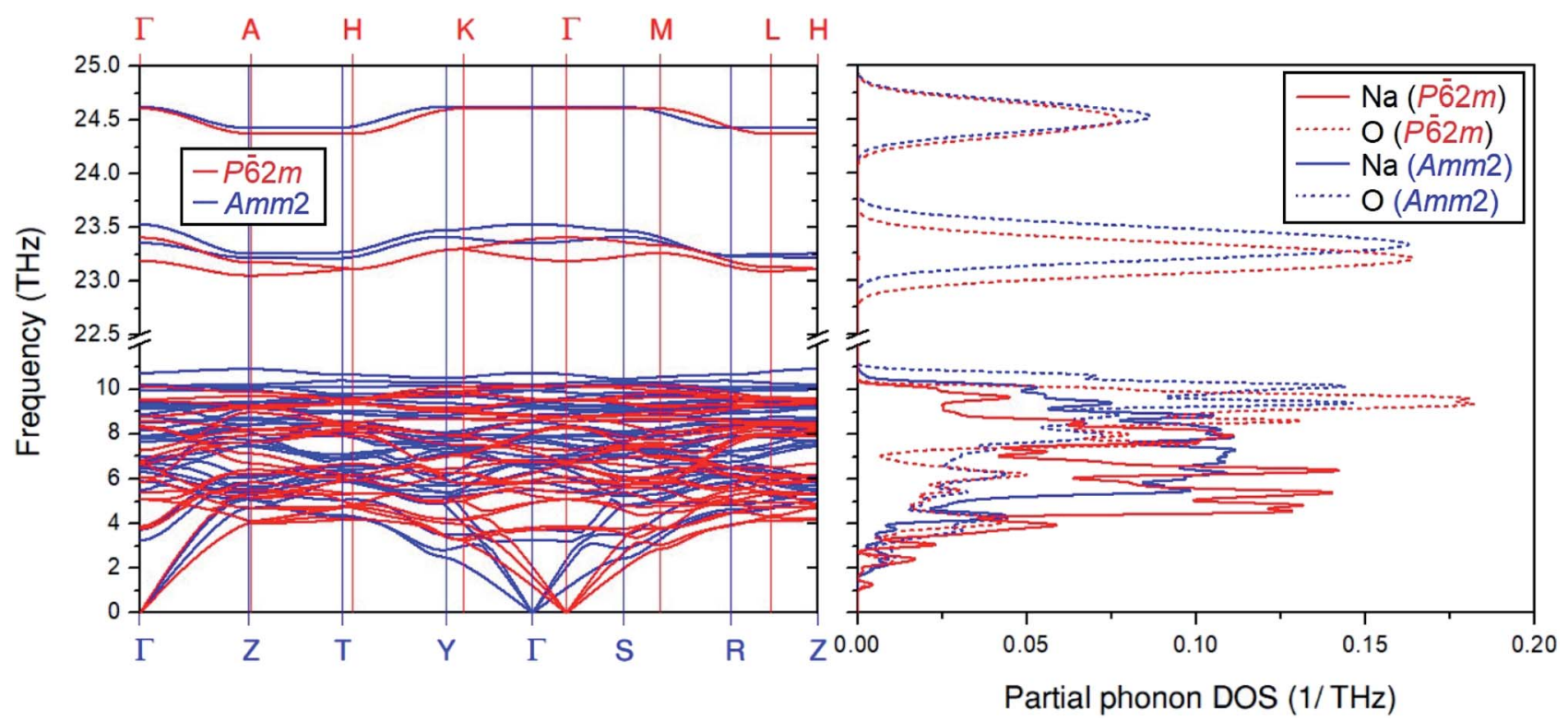

Fig. 2 The overlay plot of the phonon dispersion curves for the primitive Amm2 and the $P 62 m$ structures and their overlay plot of the partial phonon density of states. The blue and red lines represent the primitive Amm2 and the $P 62 \mathrm{~m}$ structures, respectively. 
compound, while $H(\mathrm{Na})$ and $H(\mathrm{O})$ are the enthalpy of $\mathrm{Na}$ and solid $\mathrm{O}$, respectively. The findings suggest that $\mathrm{Na}_{2} \mathrm{O}_{2}$ is more stable with increasing pressure against decomposition into $\mathrm{Na}$ and $\mathrm{O}_{2}$ (Fig. 3(a)). Similarly, the enthalpies of $\mathrm{Na}_{2} \mathrm{O}+\mathrm{O}, \mathrm{NaO}_{2}+$ $\mathrm{Na}$, and $(2 / 3) \mathrm{Na}_{2} \mathrm{O}+(2 / 3) \mathrm{NaO}_{2}$ at the same pressures are higher than that of $\mathrm{Na}_{2} \mathrm{O}_{2}$, and these enthalpy differences become greater with elevated pressure (Fig. 3(b)). These suggest that $\mathrm{Na}_{2} \mathrm{O}_{2}$ is still stable against decomposition over the pressure range studied.

In order to investigate the structural phase transitions in $\mathrm{Na}_{2} \mathrm{O}_{2}$ under high pressure, the enthalpy differences of the various structures versus pressure were calculated (Fig. 4). The findings revealed that the enthalpies of the Amm2 structure have lower values than those of the $P \overline{6} 2 \mathrm{~m}$ structure over the pressure range with values of $2 \mathrm{meV} \mathrm{f.u} .^{-1}$ at $0 \mathrm{GPa}$ and $1.6 \mathrm{meV}$ f.u. ${ }^{-1}$ at $20 \mathrm{GPa}$. By comparing the total energy $(E)$ and the work done $(P V)$, we found that all $E$ of the Amm2 structure are lower than those of the $P \overline{6} 2 \mathrm{~m}$ structure, whereas its $P V$ values are higher (Fig. S8, ESI $\dagger$ ). This implies that the $P V$ plays a key role as a driving force for the high-pressure structural phase transition of the Amm 2 structure at $0 \mathrm{~K}$. However, the Amm2 structure becomes higher enthalpy than the $P 2_{1} / c$ structure at $22 \mathrm{GPa}$ (Fig. 4). The lower enthalpy of the $P 2_{1} / c$ structure is maintained in the short pressure range of $22-28 \mathrm{GPa}$ because the Pbam structure becomes a lower enthalpy structure at $28 \mathrm{GPa}$. There was no imaginary phonon frequency in the phonon dispersion curve of the $P 2_{1} / c$ structure at $22 \mathrm{GPa}$ and the Pbam structure at $28 \mathrm{GPa}$, which satisfies the dynamical stability (Fig. S2(a) and (c), ESI $\dagger$ ). Thus, the first structural phase transition is predicted at $22 \mathrm{GPa}$, the Amm2 structure transforming to the $P 2_{1} / c$ structure, and the second structural phase transition is predicted at $28 \mathrm{GPa}$, the $P 2_{1} / c$ structure transforming to the Pbam structure. The transition pressure at $28 \mathrm{GPa}$ is the same value as predicted by Deng et al. ${ }^{\mathbf{1 0}}$
To investigate the driving force for the structural phase transitions, the changes of lattice constants and volume for the $A m m 2, P 22_{1} / c$, and Pbam structures at high pressures were considered (Fig. S9, ESI $\dagger$ ). At the first transition pressure of $22 \mathrm{GPa}$, the lattice constants $(b, c)$ and volume $(V)$ of the $P 2_{1} / c$ structure collapsed at $\gamma=141.583^{\circ}$ by about $31.4,37.1$, and $10.0 \%$ compared to the Amm 2 structure, respectively. However, the lattice constant $a$ was extended by about $11.8 \%$. This suggests that the driving force at $22 \mathrm{GPa}$ strongly affects the $b c$ plane. At the second transition pressure of $28 \mathrm{GPa}, a, b$, and $c$ of the Pbam structure collapsed by about $21.8,0.0$, and $20.4 \%$ compared to the $P 2_{1} / c$ structure at $\gamma=141.483^{\circ}$, respectively. Although there was no collapse in the $b$-axis and volume, the fact that the $a$-axis collapsed rather than the $c$-axis leads to the alignment of the peroxide groups on the (001) plane of the Pbam structure (Fig. S11(d), ESI $\dagger$ ).

Considering the peroxide groups' (O-O single bonds) alignment in each phase of $\mathrm{Na}_{2} \mathrm{O}_{2}$ (Fig. S11, ESI $\dagger$ ), the peroxide groups in the Amm2 structure aligned in parallel with the [100] direction, whereas they aligned in parallel with the [001] direction in the $P \overline{6} 2 \mathrm{~m}$ structure. In the case of the $P 2_{1} / c$ structure at $22 \mathrm{GPa}$, the peroxide groups tilted with an angle of $\sim 24^{\circ}$ with respect to the $a b$-plane and deviated from the [100] direction by $\sim 39^{\circ}$. As for the Pbam structure at $28 \mathrm{GPa}$, the peroxide groups aligned on the (001) plane but deviated from the [100] direction by $\sim 52^{\circ}$. Moreover, at $300 \mathrm{GPa}$, the peroxide group alignment was not different from that at $28 \mathrm{GPa}$. This suggests that the alignment of the peroxide groups for each phase is significantly different, which may affect the thermodynamic stability of these structures.

3.1.2 Phase stabilities at high pressures and temperatures. To verify the thermodynamic stability for the predicted structures of $\mathrm{Na}_{2} \mathrm{O}_{2}$, the QHA was employed to calculate their Gibbs free energies at various pressures up to $300 \mathrm{GPa}$ and various (a)

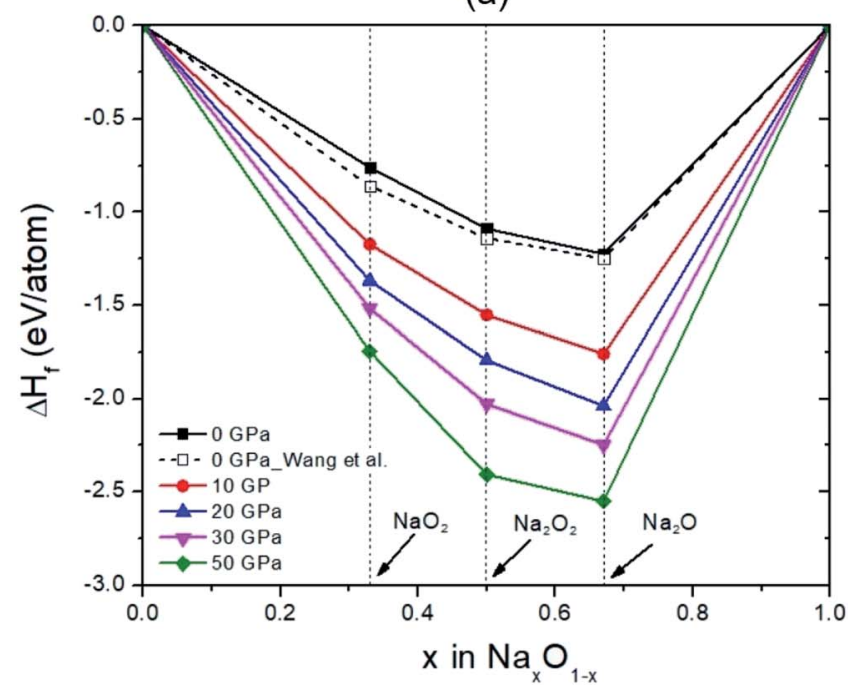

(b)

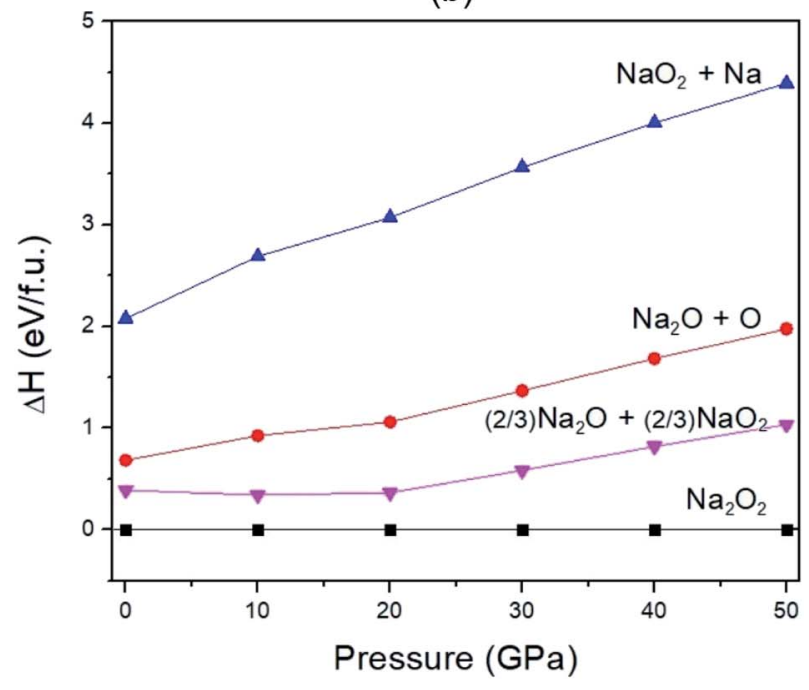

Fig. 3 (a) Predicted formation enthalpy of $\mathrm{Na}-\mathrm{O}$ compounds at different pressures with respect to decomposition into $\mathrm{Na}$ and $\mathrm{O}_{2}$. Dashed and solid lines connecting the data points denote the convex hull. The white squares represent the results predicted by Wang et al. ${ }^{35}$ (b) Relative enthalpy of the decomposition with respect to $\mathrm{Na}_{2} \mathrm{O}_{2}$ in the pressure range of $0-50 \mathrm{GPa}$. 
(a)

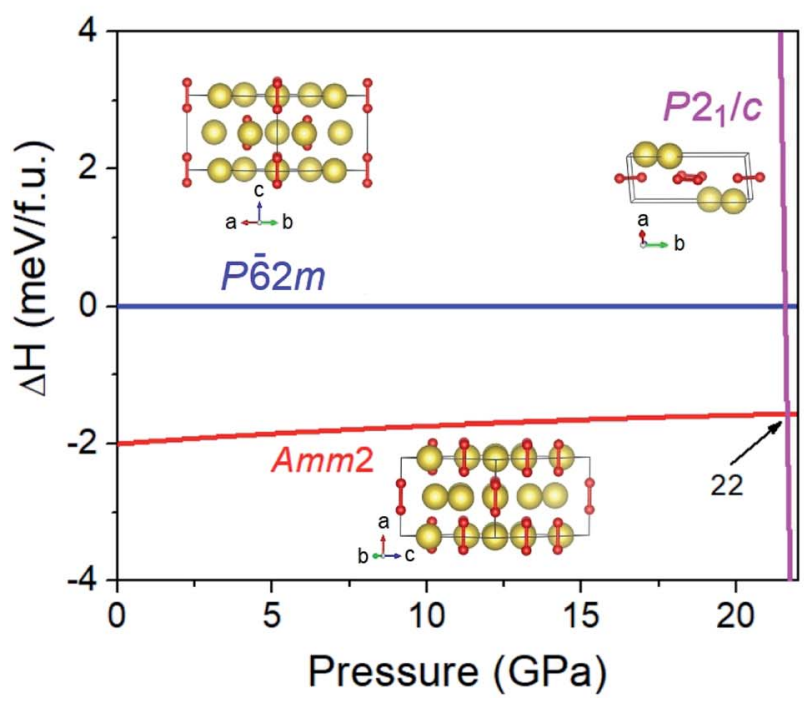

(b)

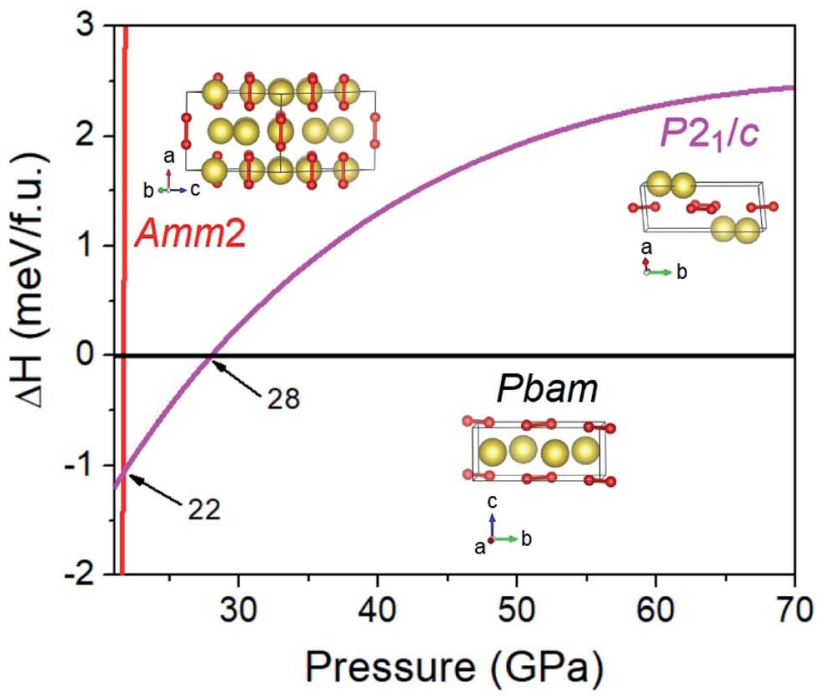

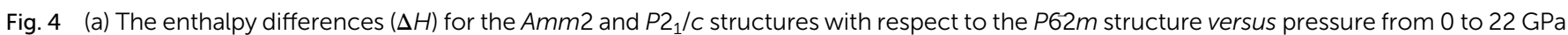

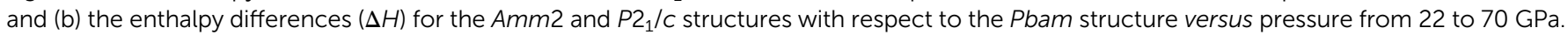
Large and small spheres in all crystal structures represent the $\mathrm{Na}$ and $\mathrm{O}$ atoms, respectively.

temperatures up to $600 \mathrm{~K}$. The findings showed that the Gibbs free energies decrease with increasing temperature, whereas they increase with increasing pressure (Fig. S12, ESI $\dagger$ ). Moreover, the Gibbs free energy changes of the Amm2 structure with respect to the $P \overline{6} 2 \mathrm{~m}$ structure at $0,5,10,15$, and $20 \mathrm{GPa}$ have higher values than those of the $P \overline{6} 2 m$ structure at the transition temperature around $120,125,185,200$, and $75 \mathrm{~K}$, respectively (Fig. 5(a)). This suggests that the $P \overline{6} 2 \mathrm{~m}$ structure becomes thermodynamically stable at these conditions. That is why the $P \overline{6} 2 \mathrm{~m}$ structure has been found to be stable at room temperature by previous measurements. ${ }^{5,6}$ Similarly, at $22 \mathrm{GPa}$, the $P 2_{1} / c$ structure at below $105 \mathrm{~K}$ has the lowest free energy compared to the other structures, while the Pbam structure becomes the lowest free energy structure in the temperature range from 105 to $600 \mathrm{~K}$ (Fig. S12(b), ESI $\dagger$ ). Moreover, at $28 \mathrm{GPa}$, the Pbam structure is thermodynamically stable in the temperature range of $0-600 \mathrm{~K}$ (Fig. S12(c), ESI $\dagger$ ). Based on these findings, the predicted $P-T$ phase diagram of $\mathrm{Na}_{2} \mathrm{O}_{2}$ was constructed as shown in Fig. 5(b).

As for the $P \overline{6} 2 m$ structure, although there was no difference in the $P \overline{6} 2 m$ structures at $0 \mathrm{~K}$ and ambient pressure that were obtained from the AIRSS searches and the relaxed Tallman's/ (a)

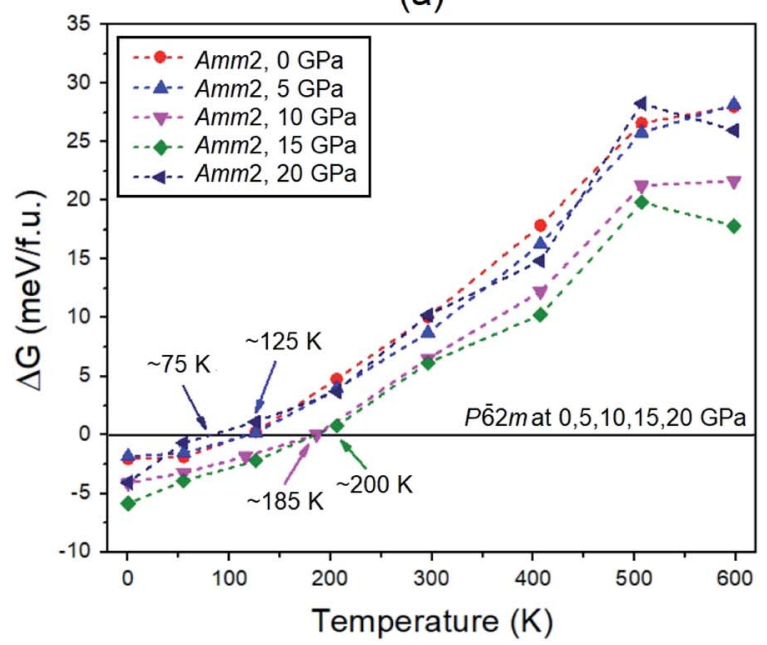

(b)

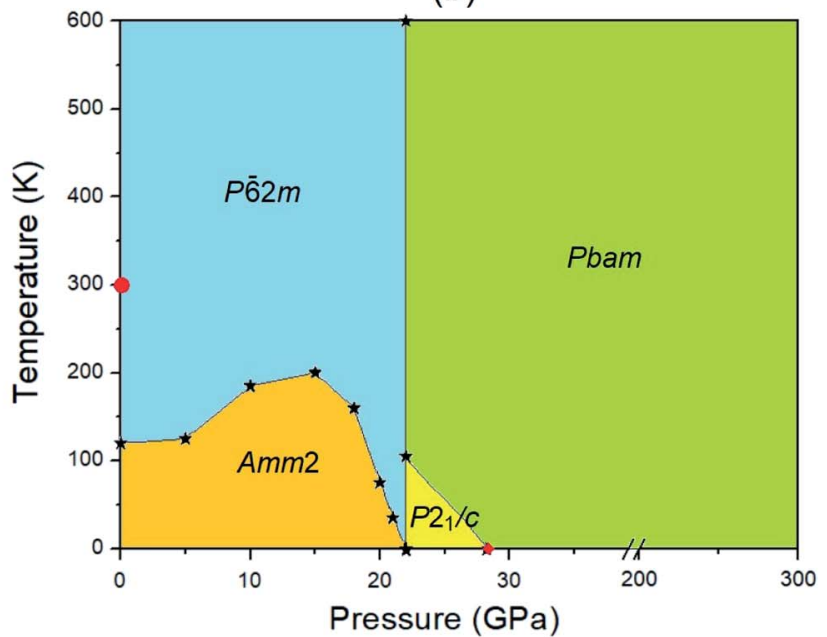

Fig. 5 (a) Gibbs free energy changes of the Amm2 structure compared to the $P 62 m$ structure at the same pressures $(0,5,10,15$, and $20 \mathrm{GPa})$ in the temperature range of $0-600 \mathrm{~K}$. (b) The predicted phase diagram of $\mathrm{Na}_{2} \mathrm{O}_{2}$ in the pressure range from 0 to $300 \mathrm{GPa}$ and at temperatures up to $600 \mathrm{~K}$. The circle indicates the $P 62 \mathrm{~m}$ structure at ambient conditions measured by Tallman et al. ${ }^{5}$ and Föppl. ${ }^{6}$ The diamond represents the Pbam structure predicted by Deng et al. ${ }^{10}$ 
Föppl's structures, there was a small significant atomic displacement of the Na1 and $\mathrm{Na} 2$ atoms between the Tallman's and Föppl's structures at room temperature (Fig. S13, ESI†) as discussed before. The consequence is the small difference in the XRD pattern between both structures (Fig. S14, ESI $\dagger$ ). In particular, the intensity of the (100) peak of Tallman's structure is higher than that of Föppl's structure by about 2 percent. These suggest that Tallman's and Föppl's structures observed at room temperature are slightly different. To identify the most stable structure at elevated temperature, we calculated the Gibbs free energy for the relaxed Tallman's and Föppl's structures at ambient pressure as a function of temperature up to 600 K. The findings revealed that the free energy of Tallman's structure is not different to that of Föppl's structure in the temperature range of $0-45 \mathrm{~K}$, yet it becomes lower than that of Föppl's structure by $\sim 1.7$ and $4 \mathrm{meV}^{\text {f.u. }}{ }^{-1}$ at 300 and $550 \mathrm{~K}$, respectively (Fig. S15, ESI $\dagger$ ). Theoretically, this suggests that Tallman's structure is likely favored over Föppl's structure at higher temperatures. However, the free energy differences are very small and are beyond experimental resolution. Therefore, Tallman's and Föppl's structures should be considered to be similar.

\subsection{Elastic behavior at high pressures}

The present work determined the elastic constants, elastic anisotropy, and elastic moduli of the Amm 2 and $P \overline{6} 2 m$ structures in order to analyze the elastic properties of both structures at high pressures. In addition, the elastic stabilities for both structures were also verified. Theoretically, the hexagonal $P \overline{6} 2 \mathrm{~m}$ structure has five independent elastic constants: $C_{11}, C_{33}, C_{44}$, $C_{12}$, and $C_{13}$, while the orthorhombic Amm2 structure has nine independent elastic constants: $C_{11}, C_{22}, C_{33}, C_{44}, C_{55}, C_{66}, C_{12}$, $C_{13}$, and $C_{23}$. The elastic stability conditions for hexagonal and orthorhombic crystal systems under hydrostatic pressure $(P)$ are given by $B_{11}>\left|B_{12}\right|, 2 B_{13}{ }^{2}<B_{33}\left(B_{11}+B_{12}\right)$, and $B_{44}>0$ for the hexagonal case and $B_{11}>0, B_{44}>0, B_{55}>0, B_{66}>0, B_{11} B_{22}>$ $2 B_{12}{ }^{2}, B_{11} B_{22} B_{33}+2 B_{12} B_{13} B_{23}-B_{11} B_{23}{ }^{2}-B_{22} B_{13}{ }^{2}-B_{33} B_{12}{ }^{2}>$ 0 for the orthorhombic case, where $C_{i j}$ and $B_{i j}$ are the elastic constants and elastic stiffnesses, respectively. ${ }^{28}$ The $B_{i j}$ are calculated from the expressions as the following: $B_{i i}=C_{i i}-P$, $B_{12}=C_{12}+P, B_{13}=C_{13}+P$, and $B_{23}=C_{23}+P$, where $i=1,2,3$, $\ldots, 6 .^{29}$

The findings revealed that the elastic stiffnesses of the $P \overline{6} 2 \mathrm{~m}$ and Amm2 structures satisfy their elastic stability conditions. This indicates that both structures are mechanically stable. Moreover, the elastic stiffnesses also demonstrate the slight difference between the $P \overline{6} 2 \mathrm{~m}$ and $A m m 2$ structures at pressures from 0 to $15 \mathrm{GPa}$ (see Fig. 6). Generally, the trend is that the elastic stiffnesses increase with increasing pressure, which indicates that the $P \overline{6} 2 m$ and Amm2 structures stiffen with increasing pressure. Nevertheless, $B_{44}$ of the $A m m 2$ structure and the $\left(B_{11}-B_{12}\right) / 2$ term of the $P \overline{6} 2 m$ structure have decreasing trends with elevated pressure. This leads to elastic instability for the $P \overline{6} 2 m$ structure when $B_{12}$ exceeds $B_{11}$, which called tetragonal shear instability. ${ }^{30}$ Likewise, the Amm2 structure has shear instability when $B_{44}$ is equal to zero. Using linear approximation, the elastic instability of both structures was found at around $35 \mathrm{GPa}$, which is higher than the predicted transition pressure of $22 \mathrm{GPa}$. However, elastic instability occurring at a higher pressure than the transition pressure which satisfied the thermodynamic stability condition has also been proposed in other ionic compounds such as $\mathrm{MgO}$ and $\mathrm{CaO}$. It has been interpreted that the existence of a significant activation barrier along the phase transformation path leads to the inconsistent transition pressure. ${ }^{30}$ Interestingly, $B_{11}$ of the Amm2 structure and $B_{33}$ of the $P \overline{6} 2 \mathrm{~m}$ structure stiffen dramatically at $2 \mathrm{GPa}$ and then soften at $3 \mathrm{GPa}$ corresponding to the unusual decreasing of the interatomic distances of $\mathrm{O} 1-\mathrm{O} 1$ and $\mathrm{O} 2-\mathrm{O} 2$ at $3 \mathrm{GPa}$ (Fig. S10(a), ESI $\dagger$ ). Moreover, $B_{22}, B_{23}$, and $B_{44}$ of the Amm2 structure, and $B_{12}$ and $B_{33}$ of the $P \overline{6} 2 \mathrm{~m}$ structure at $9 \mathrm{GPa}$ also soften slightly. This suggests that the Amm2 structure has more (a)

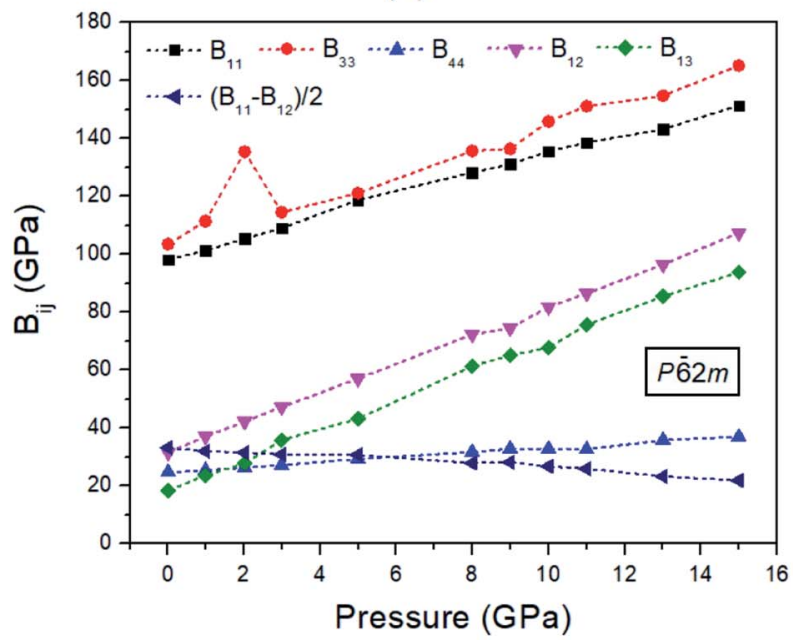

(b)

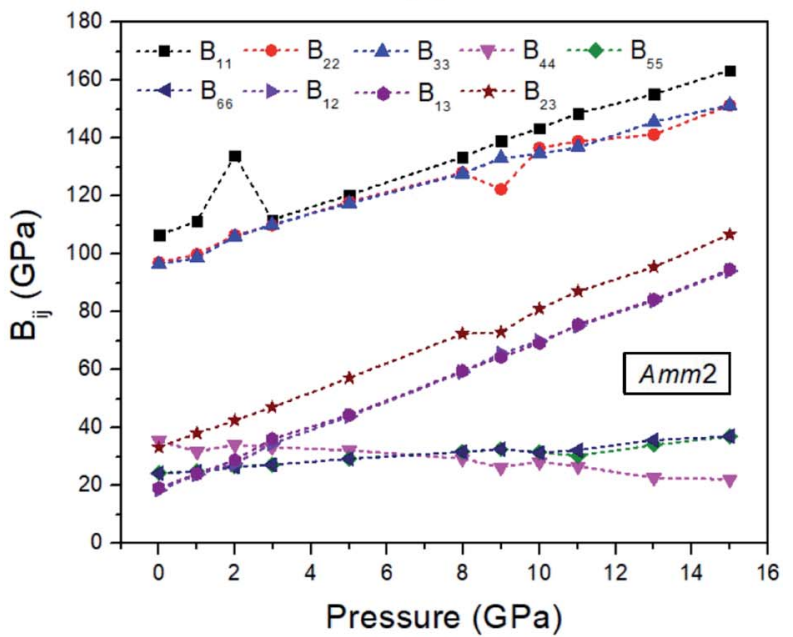

Fig. 6 The elastic stiffnesses $\left(B_{i j}\right)$ of two crystal structures at pressures ranging from 0 to $15 \mathrm{GPa}$ for: (a) the $P 62 m$ structure and (b) the Amm2 structure. 
elastic deformation than the $P \overline{6} 2 m$ structure, which may lead to thermodynamic instability at the transition temperature.

To quantify the effect of pressure on the elastic anisotropy of the crystal structures, the universal elastic anisotropy index $\left(A^{\mathrm{U}}\right)$ of the $A m m 2$ and the $P \overline{6} 2 m$ structures were calculated using eqn (2). At ambient pressure, the $A^{\mathrm{U}}$ of the $A m m 2$ and $P \overline{6} 2 m$ structures were slightly different with values of 0.28 and 0.24 , respectively (see Fig. S16, ESI $\dagger$ ). The $A^{\mathrm{U}}$ of the $P \overline{6} 2 m$ structure corresponds to that of 0.22 calculated by the previous study ${ }^{31} \mathrm{At}$ elevated pressure, the $A^{\mathrm{U}}$ of both structures decreased up to $8 \mathrm{GPa}$, and then it became increasing at around $9 \mathrm{GPa}$. Moreover, $A^{\mathrm{U}}$ significantly increased at $2 \mathrm{GPa}$ and drastically decreased at $3 \mathrm{GPa}$, which is consistent with the changes of the elastic stiffnesses at 2 and $3 \mathrm{GPa}$. Higher values of $A^{\mathrm{U}}$ indicate increasing elastic anisotropy, and when $A^{\mathrm{U}}=0$ the structure is a locally isotropic crystal. ${ }^{19}$ Thus, the findings suggest that pressure induced the changes of elastic anisotropy in the Amm2 and $P \overline{6} 2 m$ phases of $\mathrm{Na}_{2} \mathrm{O}_{2}$, and their $A^{\mathrm{U}}$ are very close to that of a locally isotropic crystal with $A^{\mathrm{U}}=0.03$ at $9 \mathrm{GPa}$, similar to other hexagonal crystals (e.g., $\mathrm{Ru}, \alpha-\mathrm{Hf}$, and $\alpha-\mathrm{Gd}) .{ }^{19}$

In addition, the calculated bulk and shear moduli of the $A m m 2$ and $P \overline{6} 2 m$ structures at ambient pressure are shown in Table S2, ESI. $\uparrow$ The findings demonstrated that the bulk and shear moduli of the Amm 2 and $P \overline{6} 2 \mathrm{~m}$ structures at ambient pressure are slightly different. It is known that the elastic moduli calculated by LDA are usually overestimates, whereas those of GGA are underestimates. ${ }^{32}$ Based on this reason, our results suggest that the measured bulk $(K)$ and shear moduli $(G)$ at ambient pressure should be $K=49.15-64.55 \mathrm{GPa}$ and $G=$ 30.49-39.29 GPa for the Amm2 structure, and $K=48.95-$ 64.73 GPa and $G=29.01-37.88 \mathrm{GPa}$ for the $P \overline{6} 2 \mathrm{~m}$ structure, respectively. Compared to the bulk moduli $\left(B_{0}\right)$ obtained from the third-order Birch-Murnaghan equations of state, $B_{0}$ for the $A m m 2$ and $P \overline{6} 2 m$ structures were 49.25 and $49.26 \mathrm{GPa}$, respectively. These values are in excellent agreement with the previous studies with the value of $49 \mathrm{GPa}$ (ref. 31) obtained from the elastic constants calculations and $56.40 \mathrm{GPa}$ (ref. 2) obtained

(a)
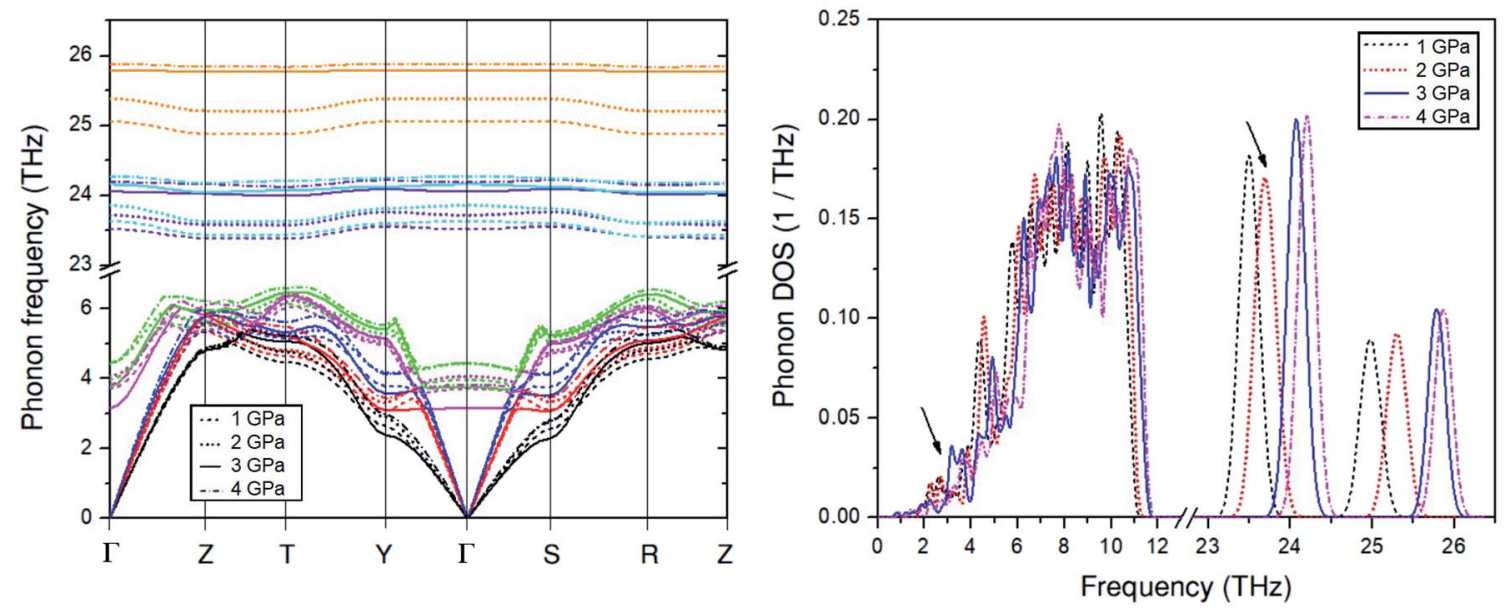

(b)
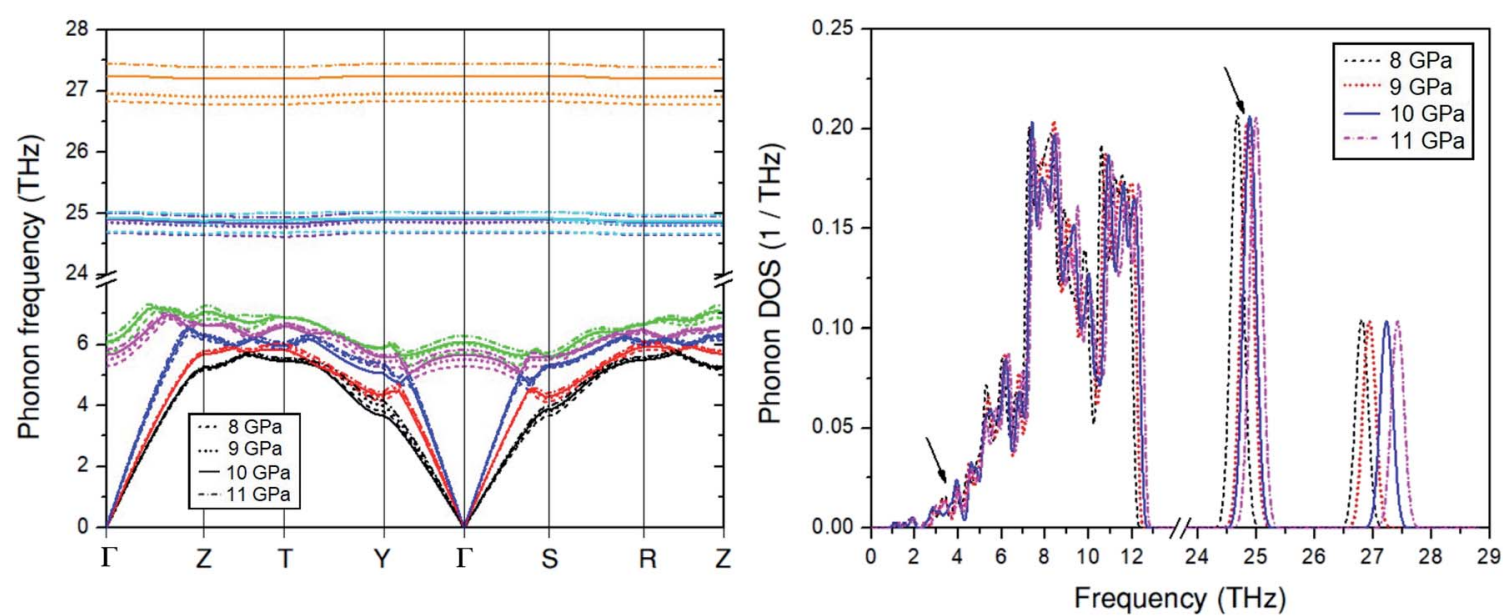

Fig. 7 Phonon dispersion curves and their phonon DOSs of the Amm2 structure: (a) at 1, 2, 3, and 4 GPa and (b) at 8, 9, 10, and 11 GPa. The phonon frequency modes in the phonon dispersion curves are distinguished by different colors. 
from the third-order Birch-Murnaghan equations of states. Based on the PBE functional using single-point energy calculations, the calculated bulk and shear moduli at room temperature were $55.82 \mathrm{GPa}$ and $31.14 \mathrm{GPa}$ for the $P \overline{6} 2 \mathrm{~m}$ structure proposed by Tallman et al., ${ }^{5}$ and $57.16 \mathrm{GPa}$ and $32.11 \mathrm{GPa}$ for the $P \overline{6} 2 \mathrm{~m}$ structure proposed by Föppl et al., ${ }^{6}$ respectively. This demonstrates the small significant difference under the upper and lower bounds on the bulk and shear moduli for the $P \overline{6} 2 m$ structure. It is possible to note that the elastic properties of Tallman's and Föppl's structures at room temperature are slightly different.

\subsection{Vibrational properties at high pressures}

Although the Amm2 and $P \overline{6} 2 m$ structures were slightly different, there was a significant difference in their vibrational properties. Using the group theory analysis of eigenvectors, the results revealed that the vibrational modes of the Amm2 structure at the $\Gamma$ point consist of $\mathrm{B}_{1}, \mathrm{~B}_{2}, \mathrm{~A}_{1}$, and $\mathrm{A}_{2}$ modes with 36 frequencies. The phonon acoustic modes are $\Gamma_{\text {act }}=B_{1}+B_{2}+$ $\mathrm{A}_{1}$, while the phonon optical modes are $\Gamma_{\mathrm{opt}}=9 \mathrm{~B}_{1}+8 \mathrm{~B}_{2}+$ $10 \mathrm{~A}_{1}+6 \mathrm{~A}_{2}$. In the case of the $P \overline{6} 2 m$ structure, the vibrational modes consist of $\mathrm{A}_{2}^{\prime \prime}, \mathrm{E}^{\prime}, \mathrm{A}_{1}^{\prime \prime}, \mathrm{A}_{1}^{\prime}, \mathrm{A}_{2}^{\prime}$, and $\mathrm{E}^{\prime \prime}$ modes with 36 frequencies as well. The phonon acoustic modes are $\Gamma_{\text {act }}=\mathrm{A}_{2}^{\prime \prime}+2 \mathrm{E}^{\prime}$, whereas the phonon optical modes are $\Gamma_{\text {opt }}=3 \mathrm{~A}_{2}^{\prime \prime}+12 \mathrm{E}^{\prime}+\mathrm{A}_{1}^{\prime \prime}+4 \mathrm{~A}_{1}^{\prime}+3 \mathrm{~A}_{2}^{\prime}+10 \mathrm{E}^{\prime \prime}$. We here predicted that there are differences in the phonon frequencies in each mode and activity in the IR and Raman spectra between the Amm 2 and the $P \overline{6} 2 m$ structures at ambient pressure (see Table $\mathrm{S} 3, \mathrm{ESI} \dagger$ ). That is, all of the phonon frequencies in the optical modes of the Amm2 structure at the $\Gamma$ point are Raman active, while for the $P \overline{6} 2 \mathrm{~m}$ structure some of them are Raman active consisting of $\mathrm{E}^{\prime}, \mathrm{A}_{1}^{\prime}$, and $\mathrm{E}^{\prime \prime}$ modes only. Similarly, only the optical modes of $\mathrm{A}_{2}^{\prime \prime}$ and $\mathrm{E}^{\prime}$ for the $P \overline{6} 2 \mathrm{~m}$ structure are IR active, whereas all of the optical modes for the Amm2 structure are IR active, except the $A_{2}$ mode. These results suggest that it is possible to observe the Amm2 structure using IR and Raman spectroscopies at temperatures below $120 \mathrm{~K}$. In addition, Table S3 (ESI $\dagger$ ) also provided the phonon frequencies in each vibrational mode, IR and Raman active modes for the $P 2_{1} / c$ structure at $22 \mathrm{GPa}$ and the Pbam structure at $30 \mathrm{GPa}$. These results might be useful for experimentally identifying the structural phase transitions at those pressures.

To discuss the effect of the elastic stiffness stiffening at $2 \mathrm{GPa}$ and softening at 3 and $9 \mathrm{GPa}$ in the Amm2 structure on phonon frequencies, we here considered the phonon dispersion curves and their phonon DOSs at 1, 2, 3, and 4 GPa and at 8, 9, 10 , and $11 \mathrm{GPa}$ as shown in Fig. 7. Moreover, we also plotted the phonon frequency shifts from that of $0 \mathrm{GPa}$ versus pressure at the $\Gamma$ and Y points in the pressure range of 0-15 GPa (Fig. 8). Normally, the phonon frequencies of a crystal structure increase with increasing pressure, which suggests the chemical bond stiffening. However, we found that three acoustic phonon frequencies $\left(\omega_{1}, \omega_{2}\right.$, and $\omega_{3}$ in Fig. $\left.8(\mathrm{~b})\right)$ below 4 THz obviously decrease at $3 \mathrm{GPa}$ around the Y and S points (Fig. 7(a)). These softening frequencies also appear in their phonon DOSs below 4 THz. Similarly, the two lowest of the optical phonon frequencies $\left(\omega_{4}\right.$ and $\omega_{5}$ in Fig. $\left.8(\mathrm{a})\right)$ near $4 \mathrm{THz}$ at the $\Gamma$ point significantly decrease at $3 \mathrm{GPa}$ (Fig. 7(a)). However, although there was no unusual change in the three highest frequencies of the optical modes $\left(\omega_{34}, \omega_{35}\right.$, and $\left.\omega_{36}\right)$ at those pressures, the increase of frequency from 2 to $3 \mathrm{GPa}$ was slightly more than that from 1 to $2 \mathrm{GPa}$ and 3 to $4 \mathrm{GPa}$, and similar to the increase of frequency from 9 to $10 \mathrm{GPa}$. Additionally there was an unusual decrease in the interatomic distances of the $\mathrm{O} 1-\mathrm{O} 1$ and the $\mathrm{O} 2-\mathrm{O} 2$ at $3 \mathrm{GPa}$ (Fig. S10(a), ESI $\dagger$ ). Furthermore, the phonon DOSs of the $\omega_{34}$ and/or the $\omega_{35}$ decrease significantly at $2 \mathrm{GPa}$ (Fig. 7(a)). This may be a consequence of the increasing of the elastic stiffness $B_{11}$ at $2 \mathrm{GPa}$. Likewise, at $10 \mathrm{GPa}$, the $\omega_{1}$ and $\omega_{3}$ below $5 \mathrm{THz}$ (a)

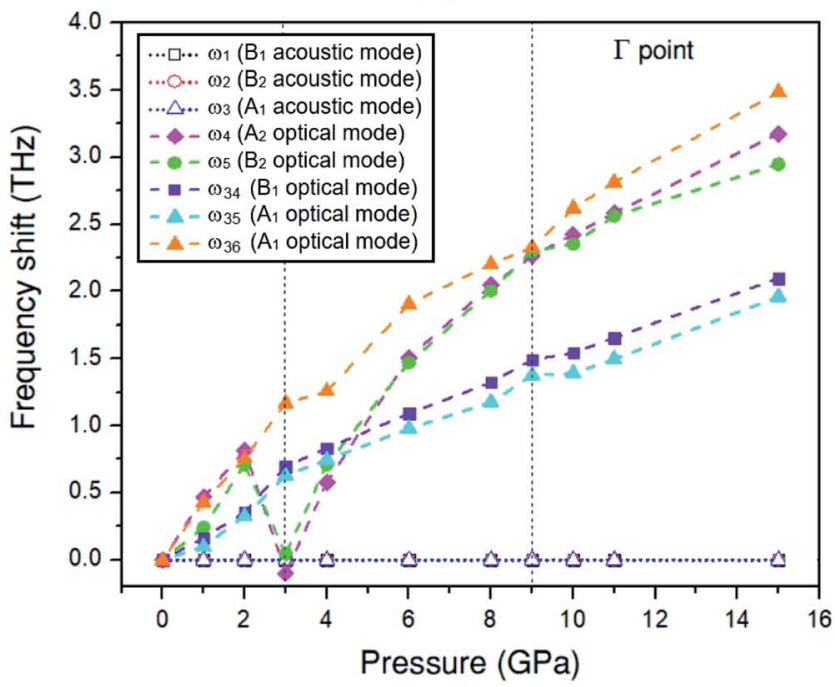

(b)

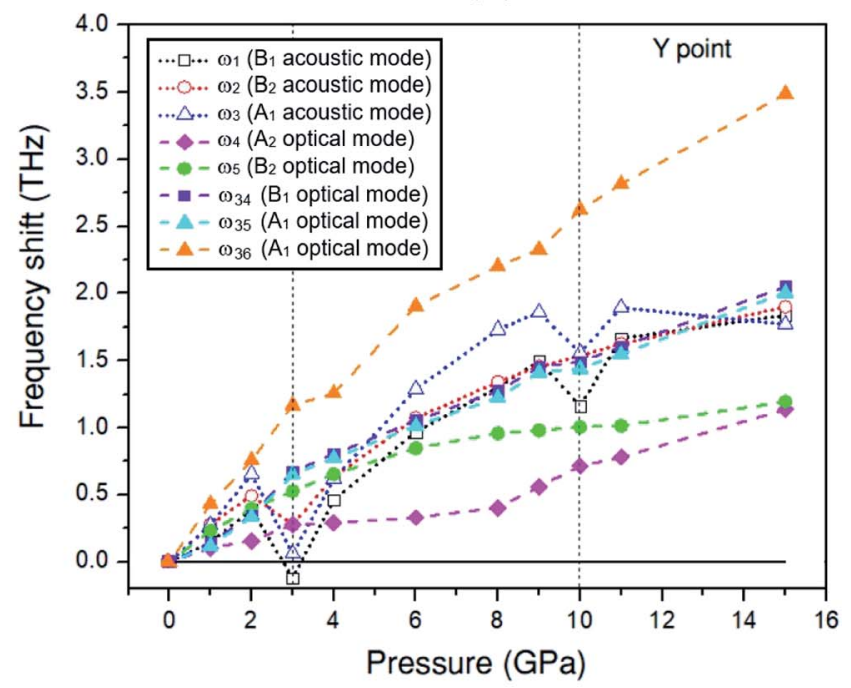

Fig. 8 The selected phonon frequency shifts versus pressure ranging from 0 to $15 \mathrm{GPa}$ for 3 frequencies of the acoustic modes and 5 frequencies of the optical modes at different $k$-points of the Amm2 structure: (a) at the $\Gamma$ point and (b) at the $Y$ points. 
obviously decrease around the Y point (Fig. 7(b)). In addition, these phonons softening result in the decreasing of the phonon free energy at 3 and $10 \mathrm{GPa}$ with respect to the trend line (Fig. S17, ESI $\dagger$ ) as well. However, this effect decreases with elevated temperature. Therefore, it is possible to note that the pressure induced the decreasing of elastic stiffness and the phonon softening resulting in the decreasing of phonon free energy at 3 and $10 \mathrm{GPa}$.

\subsection{Electronic properties and chemical bonding}

To investigate the electronic properties of $\mathrm{Na}_{2} \mathrm{O}_{2}$ under high pressure, the PBE and HSE06 functionals were employed to calculate the electronic band structures and the partial density of states (PDOSs) for the ground state of the Amm2, $P \overline{6} 2 m, P 2_{1} / c$, and Pbam structures (Fig. 9). The difference in the band structures of the Amm2 and $P \overline{6} 2 \mathrm{~m}$ structures at the $\mathrm{T} / \mathrm{H}$ points is around -0.5 and $-3.5 \mathrm{eV}$, at the $\mathrm{Y} / \mathrm{K}$ points is around -0.7 and $-4.5 \mathrm{eV}$, and at the $\mathrm{Z} / \mathrm{H}$ point is around $-0.4,-1.0,-3.3$, and $-4.0 \mathrm{eV}(A m m 2 / P \overline{6} 2 \mathrm{~m})$. Furthermore, the band structure of the Amm 2 and $P \overline{6} 2 m$ structures show indirect band gaps with the same value of $1.79 \mathrm{eV}$ calculated by the PBE functional, while the band structure of the $P 2_{1} / c$ and Pbam structures suggest direct band gaps. Compared to the HSE06 band structure, the PBE band structure is similar. However, the HSE06 functional, which usually provides a more reliable band gap than the PBE functional, predicted that the band gap of the Amm2 structure is higher than that of the $P \overline{6} 2 \mathrm{~m}$ structure by $2 \mathrm{meV}$. The PBE band gap of the $P \overline{6} 2 \mathrm{~m}$ structure is consistent with the previous study with the value of $1.76 \mathrm{eV} .^{33}$ At elevated pressure, the PBE and HSE06 band gaps of all structures have the same increasing trends (Fig. 10). However, the HSE06 band gap of the $P 2_{1} / c$ structure at $22 \mathrm{GPa}$ is higher than that of the Pbam structure at about $13 \mathrm{meV}$, which suggests a difference between the structures.

As for the PDOSs of $\mathrm{Na}$ and $\mathrm{O}$ in the Amm $2, P \overline{6} 2 m, P 2_{1} / c$, and Pbam structures (Fig. 9), they showed that the p-states of $\mathrm{O}$ mainly contribute to both the valence and conduction bands with small partial contributions from the p-states of $\mathrm{Na}$, the sstates of $\mathrm{Na}$, and a very small partial contribution from the sstates of $\mathrm{O}$. Furthermore, the direct overlap of orbitals also suggests $\sigma$ bonding of the $\mathrm{O}-\mathrm{O}$ in these structures of $\mathrm{Na}_{2} \mathrm{O}_{2}$.

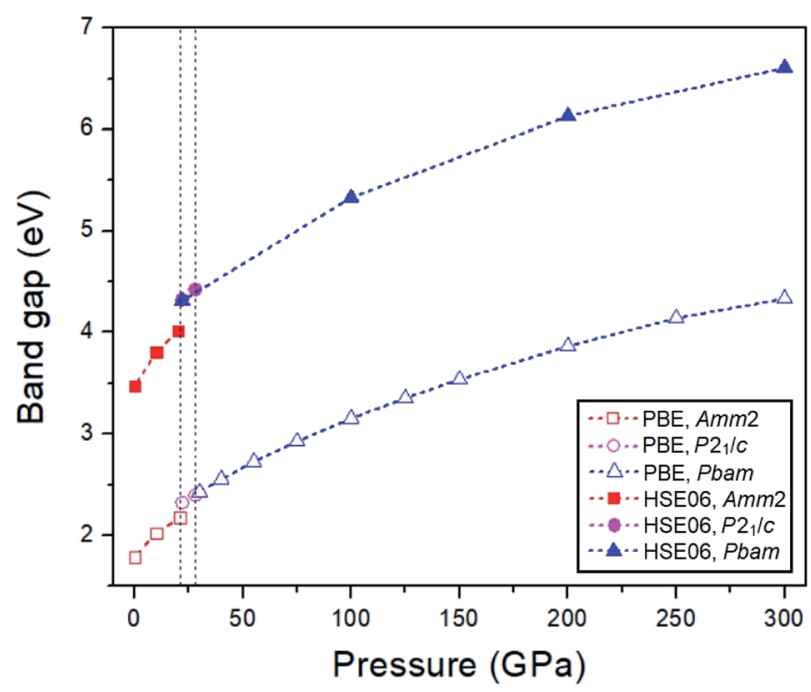

Fig. 10 The PBE and HSE06 band gaps versus pressure for the Amm2, $P 2_{1} / C$, and Pbam structures. (a) Amm2, $0 \mathrm{GPa}$
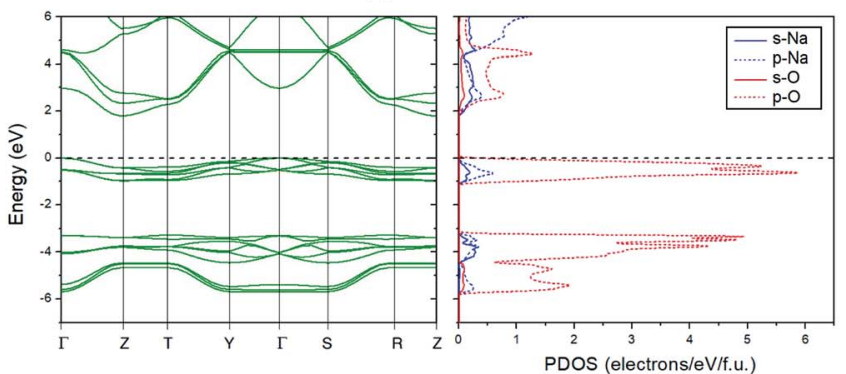

(c) $P 2{ }_{1} / c, 22 \mathrm{GPa}$
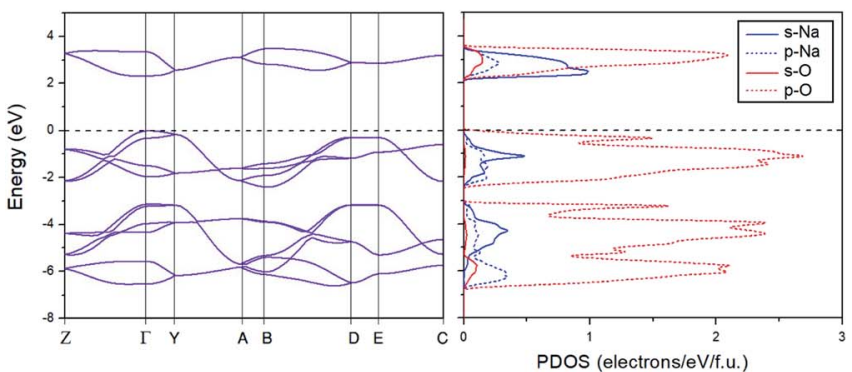

(b) $P \overline{6} 2 m, 0 \mathrm{GPa}$

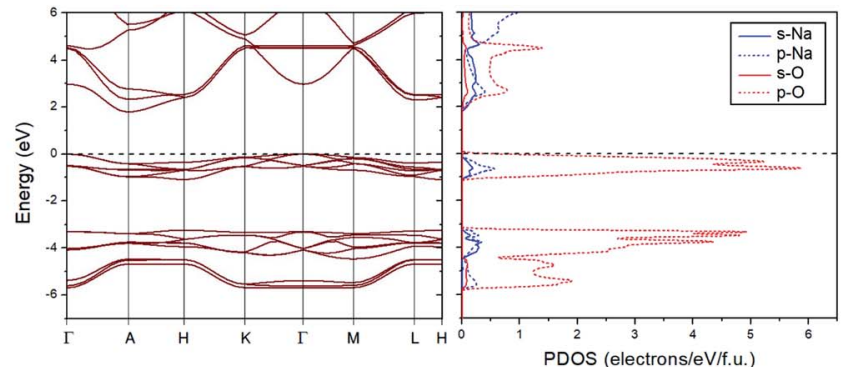

(d) Pbam, $28 \mathrm{GPa}$
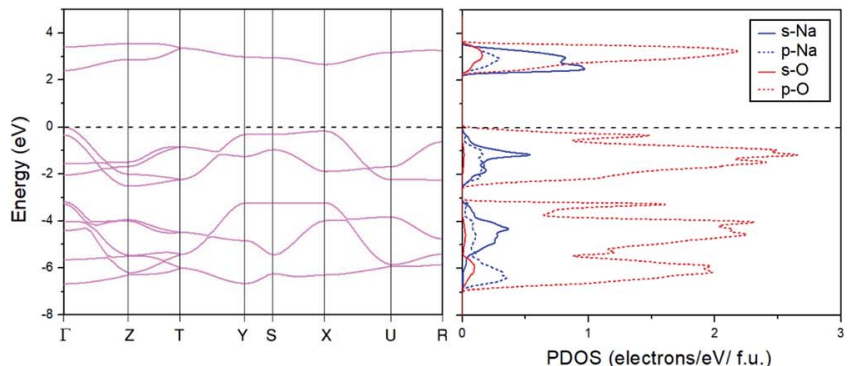

Fig. 9 Electronic band structures and their PDOSs for 4 structures at different pressures: (a) the Amm2 structure at $0 \mathrm{GPa}$, (b) the $P 62 m$ structure at $0 \mathrm{GPa}$, (c) the $P 2_{1} / c$ structure at $22 \mathrm{GPa}$, and (d) the Pbam structure at $28 \mathrm{GPa}$. 
To investigate the behavior of the $\mathrm{O}-\mathrm{O}$ bonds in the Amm2 structure of $\mathrm{Na}_{2} \mathrm{O}_{2}$ at high pressures, we calculated their ELFs in the pressure ranging from 0 to $21 \mathrm{GPa}$ (Fig. 11). The findings predicted that the ELF has a decreasing trend with elevated pressure, which suggests the decreasing of shared electrons in the $\mathrm{O}-\mathrm{O}$ bonds. Dramatically, however, the ELF turned to increase in the pressure ranges of 2-3 GPa and 9-10 GPa. The change at $3 \mathrm{GPa}$ corresponds to the softening of elastic stiffness $B_{11}$ and the softening of phonon frequencies of the acoustic modes at the $\mathrm{Y}$ point and the two-lowest frequencies of the optical modes at the $\Gamma$ point as discussed before. Similarly, the change at $10 \mathrm{GPa}$ significantly relates to the softening of phonon frequencies of the two acoustic modes at the Y point. However, the change of elastic stiffness $B_{22}$ at $9 \mathrm{GPa}$ has no correlation with the drastic increasing of the ELF at $10 \mathrm{GPa}$. Therefore, these suggest that the significantly elevated ELF at 3 and $10 \mathrm{GPa}$ significantly relate to the softening of phonon frequencies of the acoustic mode and/or the optical modes at 3 and $10 \mathrm{GPa}$. Moreover, this leads to the stiffening of the O1-O1 and the $\mathrm{O} 2-\mathrm{O} 2$ bonds at $3 \mathrm{GPa}$ as well. However, the unusual change of the $\mathrm{O}-\mathrm{O}$ bond length at $10 \mathrm{GPa}$ was not found, but there was a significant decrease of the cell angle, $\gamma$, of the primitive Amm2 structure at $10 \mathrm{GPa}$ (Fig. S9(d), ESI $\dagger$ ). In addition, dramatic changes of ELF have also been predicted in the $P 6_{3} / m m c$ structure of $\mathrm{Li}_{2} \mathrm{O}_{2}$ at 11 and $40 \mathrm{GPa} .{ }^{34}$ It was predicted that there is some charge transfer between the $\mathrm{O}$ and $\mathrm{Li}$ atoms at these pressures. However, the charge transfer may also occur in the Amm2 structure of $\mathrm{Na}_{2} \mathrm{O}_{2}$, but the marked change of the partial density of states of the $\mathrm{Na}$ and $\mathrm{O}$ atoms was not found in this work.

In addition, we also plotted the two-dimensional ELF for the Amm2 structure at ambient pressure projected onto the (110) plane, the $P 2_{1} / c$ structure at $22 \mathrm{GPa}$ projected onto the (200) plane, and the Pbam structure at 28 and $300 \mathrm{GPa}$ projected onto the (001) plane (Fig. 12). The findings showed the shared electrons in the $\mathrm{O}-\mathrm{O}$ bond and the p-orbital shape of the $\mathrm{O}-\mathrm{O}$,

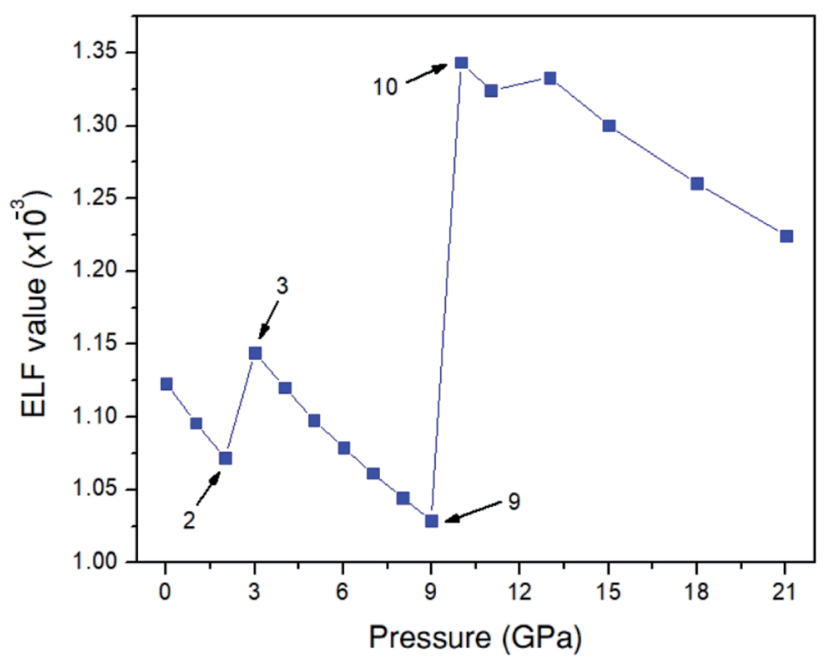

Fig. 11 Electron localization function (ELF) for the Amm2 structure in the pressure range of $0-21 \mathrm{GPa}$. (a) Amm2, $0 \mathrm{GPa}$

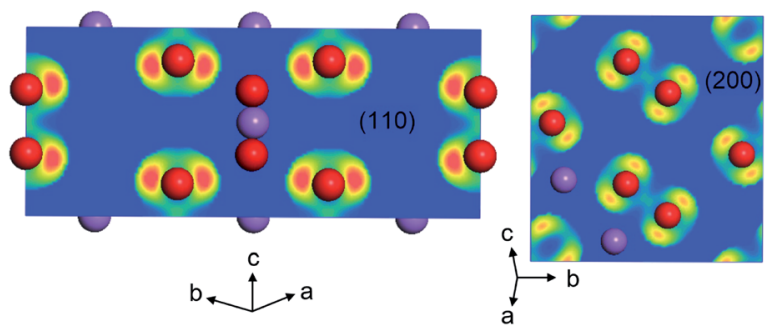

(c) Pbam, $28 \mathrm{GPa}$

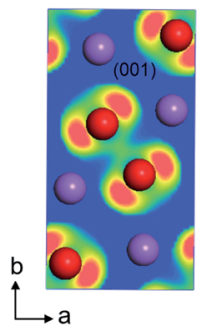

(d) Pbam, $300 \mathrm{GPa}$

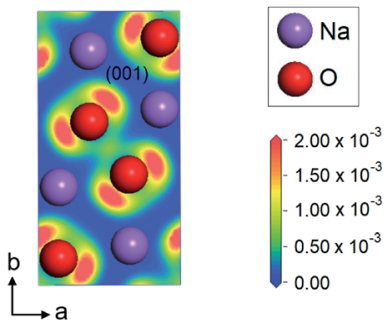

Fig. 12 Two-dimensional ELF for various structures of $\mathrm{Na}_{2} \mathrm{O}_{2}$ at different pressures: (a) the Amm2 structure at $0 \mathrm{GPa}$ projected onto the (110) plane, (b) the $P 2_{1} / c$ structure at $22 \mathrm{GPa}$ projected onto the (200) plane, and ( $c$ and d) the Pbam structure at 28 and $300 \mathrm{GPa}$ projected onto the (001) plane.

which indicates that the $\mathrm{O}-\mathrm{O}$ single bonds in these structures of $\mathrm{Na}_{2} \mathrm{O}_{2}$ remain up to $300 \mathrm{GPa}$.

\section{Conclusions}

The present work used the DFT calculations and the QHA to predict the phase stabilities of $\mathrm{Na}_{2} \mathrm{O}_{2}$ at high pressure up to $300 \mathrm{GPa}$ and temperatures up to $600 \mathrm{~K}$. The AIRSS searches were used to find the lowest enthalpy structure at $0 \mathrm{~K}$, and the dynamical and thermodynamic stabilities of the lowest free energy structure candidates were estimated using the phonon dispersion and Gibbs free energy calculations, respectively. The findings revealed the two stable structures as the lowtemperature phase of $\mathrm{Na}_{2} \mathrm{O}_{2}$. One is the Amm2 (distorted $P \overline{6} 2 \mathrm{~m}$ ) structure, which is dynamically and thermodynamically stable in the pressure range of 0-22 GPa at low temperature. However, the $P \overline{6} 2 m$ structure becomes more thermodynamically stable than the Amm2 structure at elevated temperatures. Furthermore, Tallman's structure is likely to be favored at higher temperature rather than Föppl's structure. The other is the $P 2_{1} / c$ structure, which is dynamically and thermodynamically stable in the pressure range of 22-28 GPa at extremely low temperature. Nevertheless, the Pbam structure becomes the favored structure instead of the $P 2_{1} / c$ structure at elevated temperatures. Moreover, the AIRSS searches also revealed the high-temperature phase candidates of $\mathrm{Na}_{2} \mathrm{O}_{2}$-II and $\mathrm{Na}_{2} \mathrm{O}_{2}-\mathrm{Q}$, which are $14 / \mathrm{mmm}$ and Immm structures, respectively.

Interestingly, the findings suggest the softening of the elastic stiffnesses, $B_{11}$, at $3 \mathrm{GPa}$ and $B_{22}, B_{44}$, and $B_{23}$ at $9 \mathrm{GPa}$, and the decreasing of the universal elastic anisotropy index at $3 \mathrm{GPa}$. At the $\mathrm{Y}$ point, there are three phonon acoustic modes and all 
three soften at $3 \mathrm{GPa}$ and two of them soften at $10 \mathrm{GPa}$. Similarly, at the $\Gamma$ point, the two lowest phonon optical modes at $3 \mathrm{GPa}$ also soften. This leads to the decreasing of phonon free energies and the increasing of the ELF at 3 and $10 \mathrm{GPa}$. Although the $\mathrm{O}-\mathrm{O}$ bond lengths unusually decrease at $3 \mathrm{GPa}$, the unusual change of the $\mathrm{O}-\mathrm{O}$ bond length is not found at $10 \mathrm{GPa}$, while a significant decrease of the cell angle, $\gamma$, of the primitive Amm2 structure also appears. These suggest the mechanism for maintaining the stability of $\mathrm{Na}_{2} \mathrm{O}_{2}$ at high pressures.

In addition, the PBE and HSE06 band gaps for the three phases of $\mathrm{Na}_{2} \mathrm{O}_{2}$ increase with elevated pressure, whereas they have no sign of unusual change. This suggests that the softening of the elastic stiffnesses and the softening of the phonon frequencies in the Amm2 structure have no significant effect on its band gap. Moreover, the ELFs reveal the existence of the peroxide group in $\mathrm{Na}_{2} \mathrm{O}_{2}$ up to $300 \mathrm{GPa}$. Therefore, our findings provide the two stable structures at low temperature, the candidate structures for the high-temperature phases, the $P-T$ phase diagram, and related properties of $\mathrm{Na}_{2} \mathrm{O}_{2}$ at high pressures, which might be useful for investigating the thermodynamic stability of $\mathrm{Na}_{2} \mathrm{O}_{2}$ in the $\mathrm{Na}-\mathrm{O}_{2}$ battery and the oxidizing efficiency of $\mathrm{Na}_{2} \mathrm{O}_{2}$.

\section{Conflicts of interest}

There are no conflicts to declare.

\section{Acknowledgements}

This research was funded by the $90^{\text {th }}$ anniversary of Chulalongkorn University Fund (Ratchadapisek Sompoch Endowment Fund (2019)), Chulalongkorn University. This work has been partially supported by the Super SCI-IV research grant, Faculty of Science and Chulalongkorn University; Grant for Research.

\section{References}

1 N. N. Greenwood and A. Earnshaw, Chemistry of the Elements, Butterworth-Heinemann, Oxford, 2nd edn, 1997.

2 M. V. Aleinikova, Y. N. Zhuravlev and D. V. Korabelnikov, Russ. Phys. J., 2012, 55, 495-500.

3 K. Song, D. A. Agyeman, M. Park, J. Yang and Y. M. Kang, Adv. Mater., 2017, 29, 1606572.

4 E. Zurek and W. Grochala, Phys. Chem. Chem. Phys., 2015, 17, 2917-2934.

5 R. L. Tallman, J. L. Margrave and S. W. Bailey, J. Am. Chem. Soc., 1957, 79, 2979-2980.

6 H. Föppl, Z. Anorg. Allg. Chem., 1957, 291, 12-50.

7 A. Dunst, M. Sternad and M. Wilkening, Mater. Sci. Eng., B, 2016, 211, 85-93.

8 R. L. Tallman and J. L. Margrave, J. Inorg. Nucl. Chem., 1961, 21, 40-44.

9 H. A. Wriedt, Bull. Alloy Phase Diagrams, 1987, 8, 234-246.
10 N. Deng, W. Wang, G. Yang and Y. Qiu, RSC Adv., 2015, 5, 104337-104342.

11 K. Momma and F. Izumi, J. Appl. Crystallogr., 2011, 44, 12721276.

12 S. J. Clark, M. D. Segall, C. J. Pickard, P. J. Hasnip, M. J. Probert, K. Refson and M. C. Payne, Z. Kristallogr., 2005, 220, 567-570.

13 D. Vanderbilt, Phys. Rev. B: Condens. Matter Mater. Phys., 1990, 41, 7892-7895.

14 J. P. Perdew, K. Burke and M. Ernzerhof, Phys. Rev. Lett., 1996, 77, 3865-3868.

15 C. J. Pickard and R. J. Needs, J. Phys.: Condens. Matter, 2011, 23, 053201.

16 J. R. Nelson, R. J. Needs and C. J. Pickard, Phys. Chem. Chem. Phys., 2015, 17, 6889-6895.

17 F. Birch, Phys. Rev., 1947, 71, 809-824.

18 http://www.tcm.phy.cam.ac.uk/castep/documentation/ WebHelp/content/modules/castep/tskcastepcalcelastic.htm.

19 S. I. Ranganathan and M. Ostoja-Starzewski, Phys. Rev. Lett., 2008, 101, 055504.

20 R. Hill, Proc. Phys. Soc., London, Sect. A, 1952, 65, 349-354.

21 W. Frank, C. Elsässer and M. Fähnle, Phys. Rev. Lett., 1995, 74, 1791-1794.

22 https:/www.tcm.phy.cam.ac.uk/castep/documentation/ WebHelp/content/modules/castep/thcastepthermo.htm.

23 A. Siegel, K. Parlinski and U. D. Wdowik, Phys. Rev. B: Condens. Matter Mater. Phys., 2006, 74, 104116.

24 J. Heyd, G. E. Scuseria and M. Ernzerhof, J. Chem. Phys., 2003, 118, 8207-8215.

25 D. M. Ceperley and B. J. Alder, Phys. Rev. Lett., 1980, 45, 566569.

26 J. P. Perdew and A. Zunger, Phys. Rev. B: Condens. Matter Mater. Phys., 1981, 23, 5048-5079.

27 A. Jain, S. P. Ong, G. Hautier, W. Chen, W. D. Richards, S. Dacek, S. Cholia, D. Gunter, D. Skinner, G. Ceder and K. A. Persson, APL Mater., 2013, 1, 011002.

28 F. Mouhat and F.-X. Coudert, Phys. Rev. B: Condens. Matter Mater. Phys., 2014, 90, 224104.

29 G. V. Sin'ko and N. A. Smirnov, J. Phys.: Condens. Matter, 2002, 14, 6989-7005.

30 B. B. Karki, G. J. Ackland and J. Crain, J. Phys.: Condens. Matter, 1997, 9, 8579-8589.

31 M. de Jong, W. Chen, T. Angsten, A. Jain, R. Notestine, A. Gamst, M. Sluiter, C. K. Ande, S. van der Zwaag, J. J. Plata, C. Toher, S. Curtarolo, G. Ceder, K. A. Persson and M. Asta, Sci. Data, 2015, 2, 150009.

32 G. I. Csonka, J. P. Perdew, A. Ruzsinszky, P. H. T. Philipsen, S. Lebègue, J. Paier, O. A. Vydrov and J. G. Ángyán, Phys. Rev. B: Condens. Matter Mater. Phys., 2009, 79, 155107.

33 S. Yang and D. J. Siegel, Chem. Mater., 2015, 27, 3852-3860. 34 P. Jimlim, K. Kotmool, U. Pinsook, S. Assabumrungrat, R. Ahuja and T. Bovornratanaraks, Phys. Chem. Chem. Phys., 2018, 20, 9488-9497.

35 B. Wang, N. Zhao, Y. Wang, W. Zhang, W. Lu, X. Guo and J. Liu, Phys. Chem. Chem. Phys., 2017, 19, 2940-2949. 